\title{
The Impact of Mobility Models on the Consumption of Network Resources in the Internet of Things (IoT)
}

\author{
Rasha J. Al-Jarah \\ rjhaljarah@gmail.com
}

Basim M. Mahmood

Department of Computer Science

bmahmood@uomosul.edu.iq

College of Computer Sciences and Mathematics

University of Mosul, Mosul, Iraq

\section{Received on: 19/01/2020}

Accepted on: 02/02/2020

\begin{abstract}
Nowadays, the field of Internet of Things (IoT) has become a new trend and one of the most attractive areas of research. It has a wide range of applications; starting from smart devices to developing smart cities. The main issue in this kind of applications is the limitation in network resources (e.g., energy, memory, connectivity, etc.). Most of the works in the literature deal with this issue in a traditional way. For instance, developing routing protocols that find the optimal path for data forwarding. This paper looks to this issue from a different angle. In this work, we aim at testing different mobility patterns and then investigate their impact of the consumption of network resources under particular distributions and data routing protocols. The aspect we aim to investigate and measure is the amount of data exchanged, which in turn affects the power and the memory consumption of a network. We also measure two more aspects; performance stability, and data coverage area. The results show that mobility models play a significant role in the overall network performance.
\end{abstract}

Keywords: Complex Networks, Human Mobility Model, Objects Distribution, Routing Protocols.

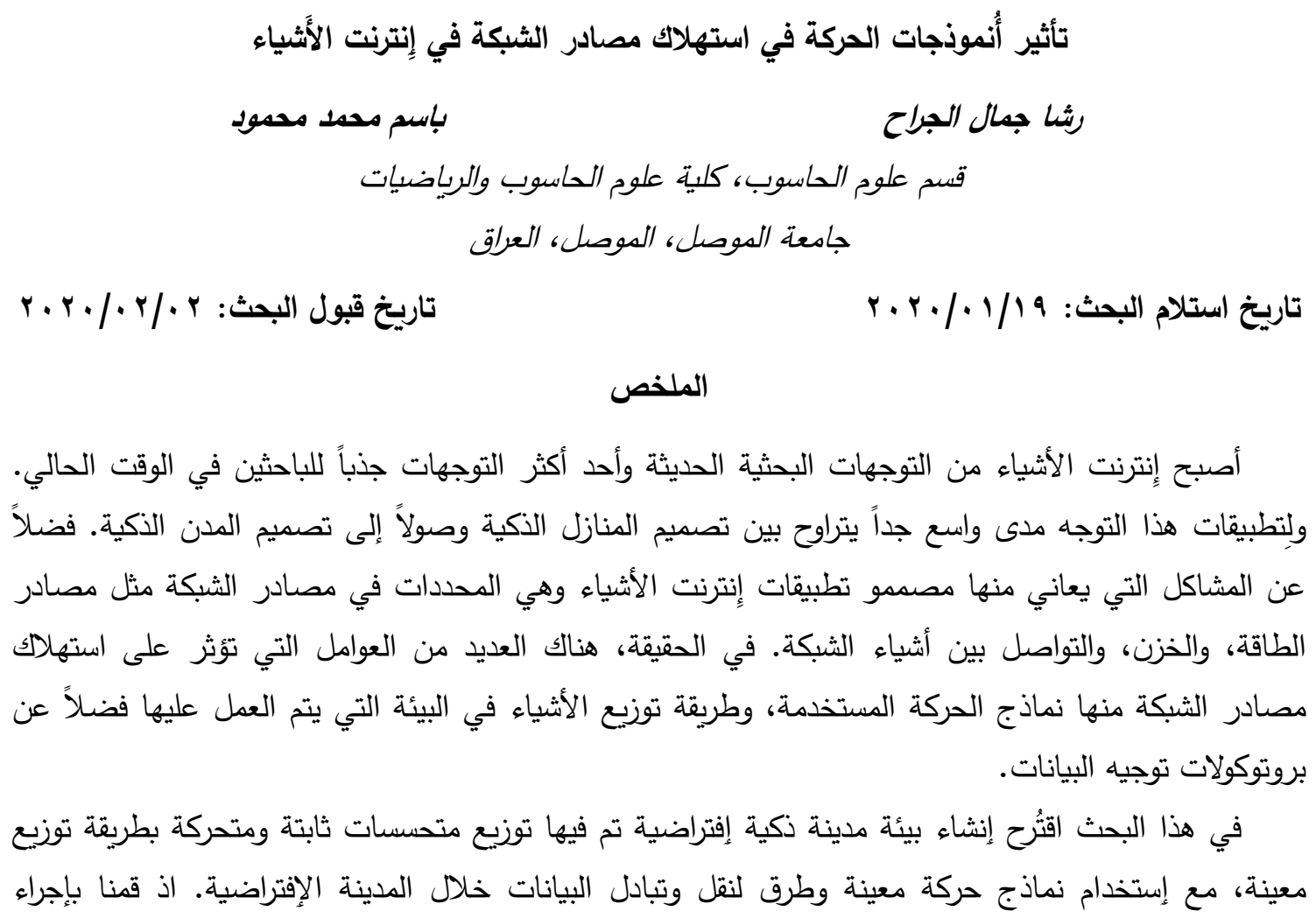


إختبارات عديدة لنماذج حركة مختلفة والتي من ضمنها: نموذج التتقل الفردي (نموذج حركة الانسان) ونموذج الاتجاهات مشتركة العلاقة ونموذج كاوجي، والتقصي عن تأثيرها في استهلاك مصادر الثبكة باستعمال توزيع معين مثل التوزيع الطبيعي والتوزيع الموحد وتوزيع الذيل الطويل، وبروتوكول معين لتوجيه البيانات مثل بروتوكول

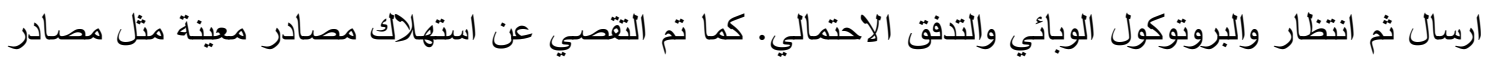
الطاقة، وكميات استهلاك البيانات. وكل تجربة هي عبارة عن مزيج من نموذج حركة وطريقة توزيع للأشياء

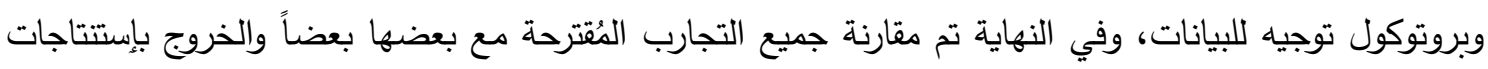

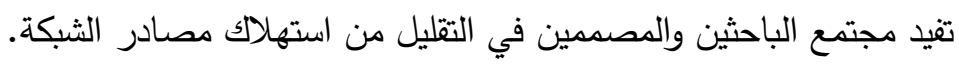
الكلمات المفتاحية: نماذج الحركة، بروتوكولات التوجياه، نموذج حركة الانسان، الثبكات المعقدة.

1. 1 - 1

يتكون إنترنت الأشياء Internet of Things (IoT) من مجموعة من الكائنات أو الأجهزة التي لها القابلية على الارتباط بالثبكة العنكبوتية. هذه الأشياء اما ثابتة؛ والتي تكون ذات موقع ثابت في الثبكة مثل متحسسات الحرائق. او متحركة؛ بمعنى ليس لها موقع ثابت وتكون متحركة مثل: أجهزة تحديد الموقع المثبتة في السيارات أو دأو عربات الإسعاف وغيرها. ويعدّ اقتران الأجهزة المستشعرة بالبشر أمراً مثيراً للاهتمام نظراً لحقيقة أن العقد ستتحرك نتيجة للحركة البشرية.

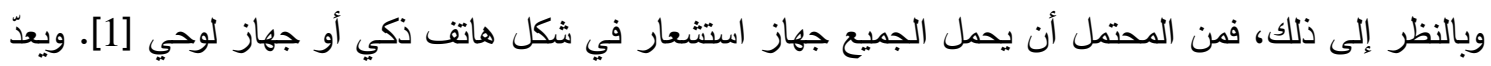

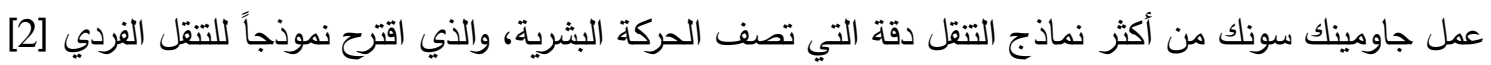

ويمكن دمج هذا النموذج في شبكات المتحسات اللاسلكية المتحركة لأغراض المحاكاة والتقييم [3]. وفي هذا البحث سوف نقوم بإجراء تجارب حول تأثير نماذج الحركة وطرائق توزيع الأشياء في استهلاك لكئك مصادر شبكة إنترنت الأشياء. وإن ما سيتم قياسه هو عدد الرسائل (Sending and Receiving Messages) التي تُتهلك في ذاكرة الأجهزة الأشياء (Memory Consumption)، والتأخير التي تستهلكه الشبكة في نشر

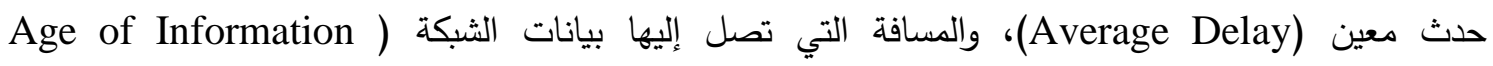

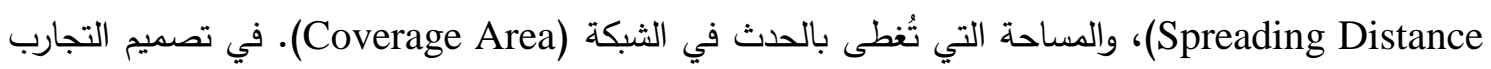

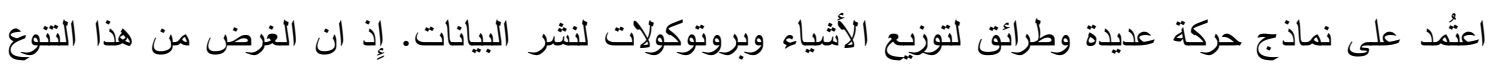

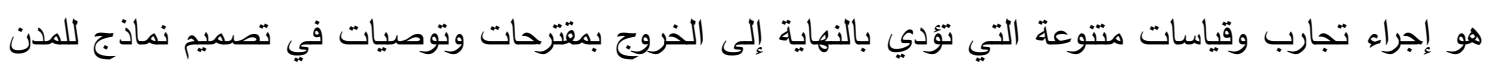

الأهداف الرئيسية لهذا البحث يمكن أن تكون على النحو التالي:

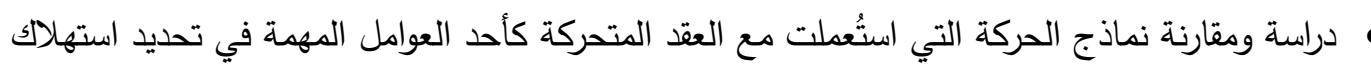
مصادر الثبكة مع طرق معيارية في هذا المجال.

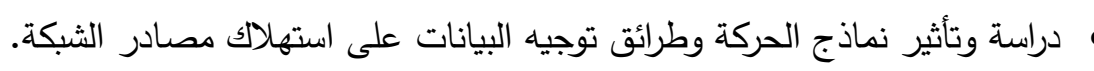

3. الأعمال السابقة

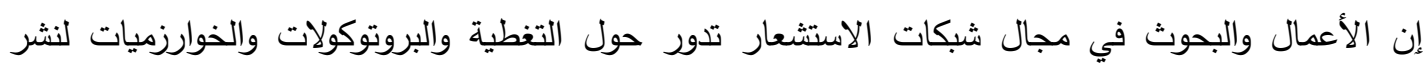
أجزة الاستشعار على النحو الأمثل [4,5]. ويتمثل الاختلاف الرئيس بين هذه الأعمال في كيفية حساب المواضع 
المطلوبة لأجهزة الاستثعار وعادةً ما يُستغل التتقل فقط لِتحقيق تكوين مثالي ثابت في بيئة استثعار موسعة، بدلاً

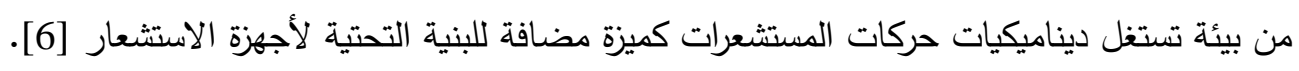
دراسة حديثة قام بها دي ميلو وآخرون [7]، اقترحت استراتيجية تسَّمى RECAST لتحليل تفاعلات الأفراد

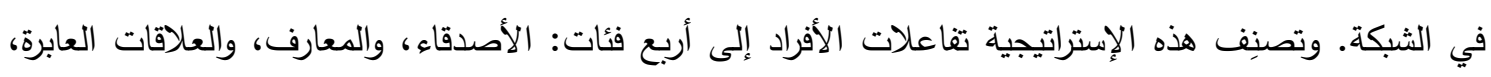

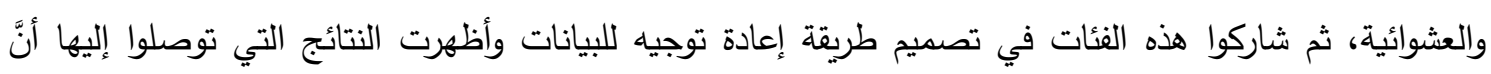

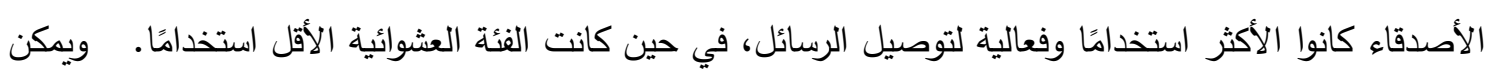

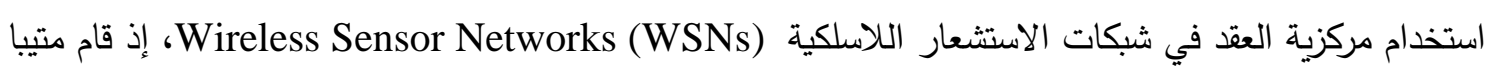
وآخرون [8] باقتراح طريقة إعادة توجيه جديدة تشّمى People-Rank، حيث يعتمد تصميمهم على خوارزمية تصنيف الصفحات وميزة مركزية العقد في الثبكة. إذ ترتبّ العقد وفقًا لمعلوماتهم الاجتماعية؛ وتُعطى العقدة وزنًا

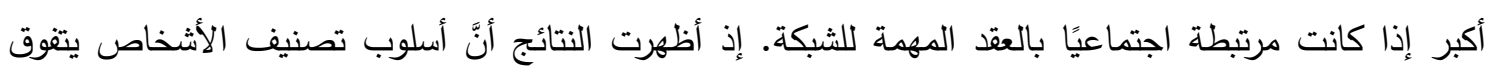
على طريقة Epidemic من حيث عدد الرسائل المتبادلة ووقت التسليم.

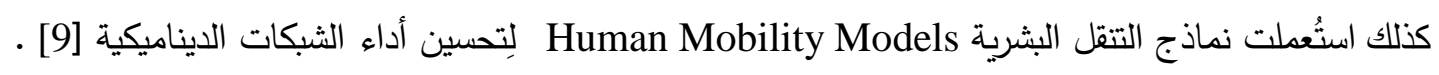
وقد أوضح توماسيني في [10] وكذلك في [3]، بأنَّ شبكات الاستشعار اللاسلكية مع أنماط الحركة الاجتماعية يمكن أن تحسن أداء شبكات الاستشعار من حيث تغطية العقد، وكذلك تسليم البيانات. لقد تم التركيز في بحثنا على معدل تسليم البيانات ومساحة التغطية ومدى استقرارية كل طريقة على التى اعتبارهم عوامل رئيسية في استهلاك الطاقة ضمن الثبكة. وبثكل أكثر تحديداً حاولنا تحسين عملية إعادة توجيه البيانات إلى أجهزة استثعار أُخرى في الثبكة، ونموذج حركة الإنسان المستخدم في هذا البحث المقترح والذي اقترحه

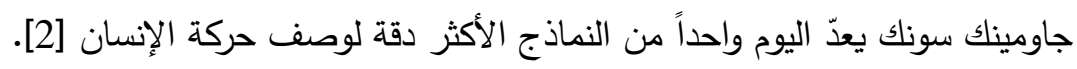

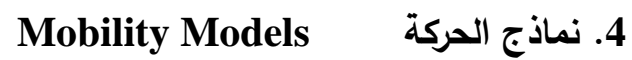

عند النظر في السياقات المذكورة آنفًا، نلاحظ أنَّ حركة أجهزة الإستثعار أكثر تعقيدًا، ولكن يمكن تفسيرها بما يسّمى نموذج التتقل Mobility Model الذي يصف حركة العقد المتتقلة Mobile Nodes وكيف أن موقعها واتجاهها وسرعتها يتغير مع مرور الوقت [9]. وتوجد أنواع عديدة من نماذج الحركة منها:

\section{Human Mobility Model 1-4 نموذج حركة الإنسان}

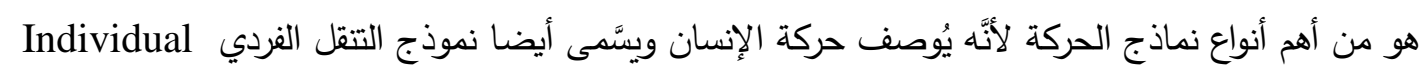

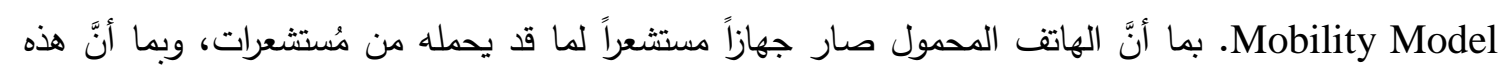

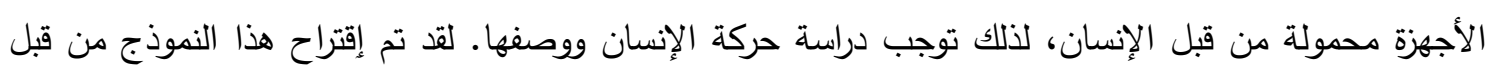
Song

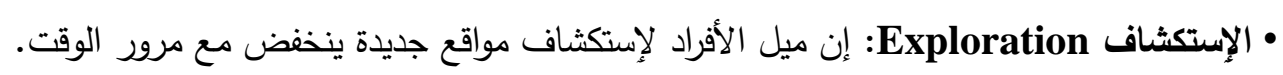
• الرجوع المفضل Preferential Return: يميل الإنسان إلى العودة إلى المواقع التي تم زيارتها سابقاً بثكل متكرر (مثل الرجوع إلى المنزل أو موقع العمل). وبسبب توفر بيانات احداثيات المواقع للبشر، ركزت الدراسات الحديثة على اقتراح نماذج تتقل تعكس حركة الانسان. اذ دَرَسَ هذه الحركة سونك وآخرون [11] الذين استكثفوا حدود القدرة على التتبؤ في الديناميكيات البشرية؛ ووجدوا إمكانية تتبؤ بنسبة 93\% في تتقلات المستخدمين وأظهروا أنّها مستقلة عن المسافة التي يغطيها 
المستخدمون بشكل منتظم حتى لو كانت أنماط السفر تختلف اختلافًا كبيرًا وهذه النتائج مهمة بشكل خاص لأنَّها تخبرنا أنَّ الحركات البشرية هي ليست عشوائية وانما ممكن أن نقول بأنها حركات عشوائية منتظمة ومن ثمَّ يمكن إستغلال قابلية التتبؤ لزيادة أداء وكفاءة بروتوكولات شبكة المُستشعرات (مثل توجيه البيانات).

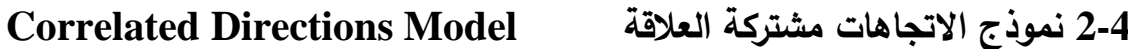

تصف نماذج التتقل بصورة عامة الموقع والسرعة والحالات الديناميكية الأخرى للأجسام المتحركة. في هذا النوع من نموذج الحركة نركز إهتمامنا على تتقل المركبات في شوارع المدينة. إذ أصبح من الواضح مدى أهمية الهرية

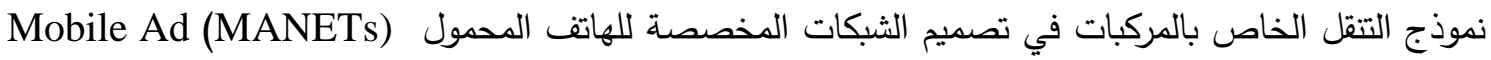
تأبر hoc Networks ، لأنه يحدد أنماط التتقل مثل الأوقات بين إتصالات المركبات مع بعضها. هذه الأوقات لها تأثير كبير على تغطية الثبكة وخصائص تأخير النقل [12,13].

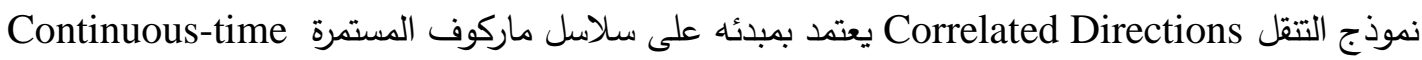
Markov chains فإن السيارة التي تتحرك في المدينة تتتمي إلى نقطة واحدة في الشبكة في وقت واحد وتتتقل إلى نقاط الثبكة

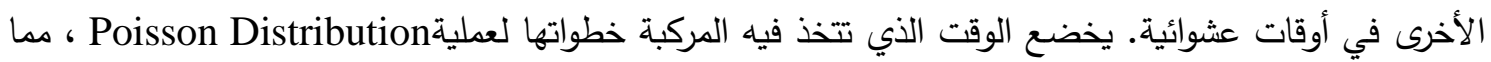
يعني أنها تتتقل من تقاطع واحد إلى تقاطع مجاور بسرعة تم إختيارها عثوائيًا. حيث يختلف إحتمال تحرك مركبة

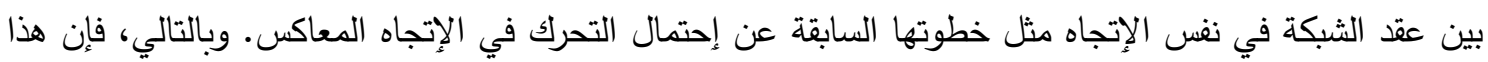

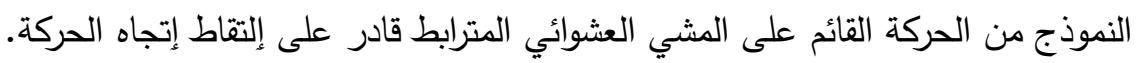

\section{Cauchy Flight Model}

3-4 نموذج كاوجي

يعدّ هذا النموذج من نماذج الحركة العشوائية التي تعتمد بمفهومها على نموذج حركة Brownian العشوائية.

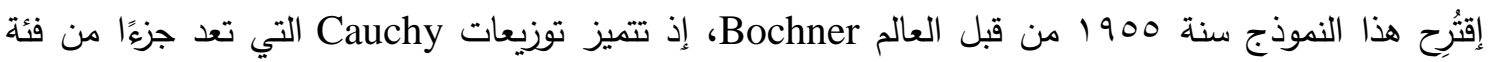

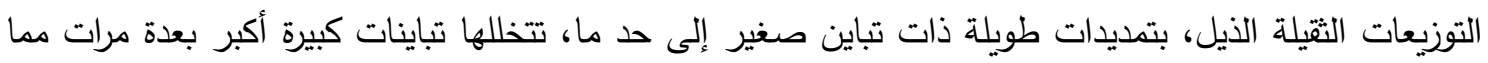
يمكن توقعه من التوزيع الطبيعي [14] كما إنه واحد من التوزيعات القليلة المستقرة والذي يمتلك دالة كثافة الإحتمال التي يمكن التعبير عنها تحليلياً.

\section{Smart City Simulator محاكي المدينة الذكية}

لِلمحاكاة واقع المدينة الذكية وتتفيذ تجارب هذا البحث نحتاج إلى محاكي يُعتمد عليه لتنفيذ التجارب. وقد اعتُمد على أَحد المحاكيات المستخدمة لهذا الغرض الذي يسمح بإجراء تجارب هذا البحث مع التتوع في تغيير إعداداته. إنَّ المحاكي الذي اعتُمد عليه في هذا البحث يسّمى بالثبكة الاجتماعية للمستثعرات (Social (SNoS) Network of Sensors) تتفيذ تجاربهم واستحصال نتائج موثوقة كون أن النتائج التي تم الحصول عليها من خلال هذا المحاكي تم نشرها في مجلات عالمية رصينة مما يؤكد موثوقية هذا المحاكي. وان مايميز هذا المحاكي أنه يجمع الكثير من مميزات

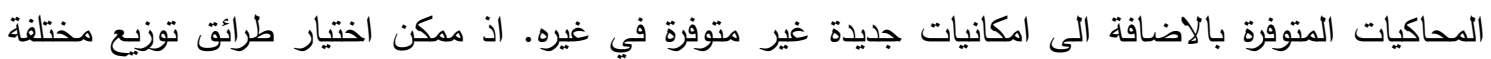
بالنسبة للمتحسسات الثابتة والمتحركة، والتحكم بعدد المتحسات الثابتة والمتحركة ايضا. واختيار نماذج حركة فئه مختلفة، وكذلك من الممكن اختيار طرائق عديدة لنقل وتوجيه البيانات بين مستشعر واخر ، والعديد من الامكانيات

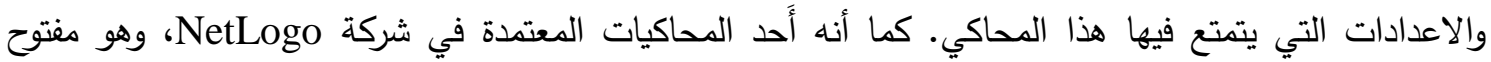


المصدر، أي يمكن إجراء التغييرات البرمجية عليه على وفق الحاجة ولقد تمت برمجة هذا المحاكي باستخدام لغة NetLogo MultiAgent Modelling Programming بالتقنيات المستخدمة في المدن الذكية (Smart Cities)، وإنترنت الأشياء (IoT). إذ يمكن تطبيق توزيع سكاني معين على الدينة التي يفترضها المحاكي وإجراء تجارب تخص بث وتوزيع البيانات، ومناطق تغطيتها، متابعة الحركة السكانية في مناطق المدينة، وتعقب أثخاص معينين داخل المدينة، ومدى انتشار معلومة معينة، ومراقبة مناطق معينة، واستثعار أحداث معينة، ومتابعة مركبات معينة، وتقصي أنماط الحركة، وتقصي مناطق التغطية

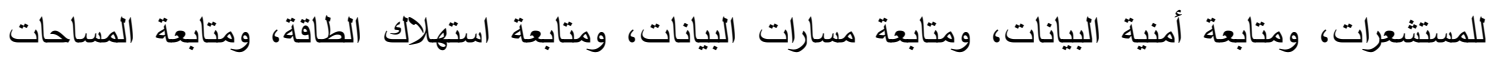

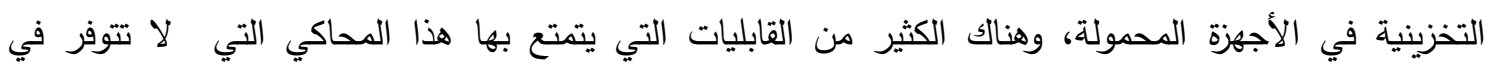
المحاكيات المعروفة.

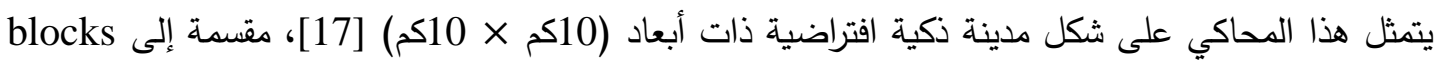
تمثل المباني والمساحات التي تعدّ محاكاة للمدن الاعتيادية بتصميمها، ويتمتع هذا المحاكي بقابلية العرض الثنائية والثلاثية الأبعاد، ويمكن أيضاً تحديد طريقة التواصل اللاسلكي ومدى الاتصال الذي يصل إلى 50 متر (WiFi). ويتميز هذا المحاكي بقابليته على إجراء إعدادات لتجارب المدن التعادئ الذكية كما يأتي: •توزيع النسبة السكانية حسب ما تتطلبه التجربة المراد تطبيقها. •تحديد نمط التوزيع السكاني، إذ يوفر المحاكي أنماط مختلفة من التوزيع حسب طبية لتبعة التوزية التيع السكاني للمدينة المراد محاكاتها.

•توزيع متحسسات استثعار ثابتة ومتحركة في المدينة (Static and Dynamic Sensors). ويمكن أيضاً توزيعها حسب التوزيعات المذكورة في النقطة السابقة.

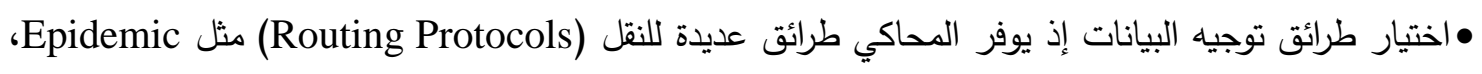
Probabilistic، PRoPHET،Spray and Wait •يمكن التحكم بمجال الاتصال لكل متحس في المدينة (communication range). ويمكن استخدام مبادئ النقل اللاسلكية المعروفة مثل (WiFi or Bluetooth). •فيما يخص حركة السكان وحركة كل فرد فإنَّ المحاكي يوفر نماذج حركة خاصة بحركة الإنسان والمعتمدة عالمياً في هذا نوع من التجارب مثل نموذج التقل الفردي (Individual Mobility Model) الذي يعذّ من أَحدث فئ نماذج الحركة وأشهرها المعتمدة لمحاكاة حركة الإنسان. •فيما يخص المتحسات الثابتة، يمكن للمحاكي اختيار Topology معينة على وفق ما يحتاج إليه الباحث في تجربته. • فيما يخص المتحسات المتحركة، يوفر المحاكي مجموعة من نماذج الحركة التي تحاكي بتصميمها نماذج حركة الأشياء.

بناءاً على ما سبق يمكن بإستخدام هذا المحاكي تصميم وتخطيط المدن الذكية، وتصميم خوارزميات بث

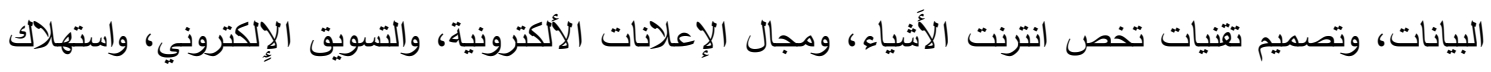

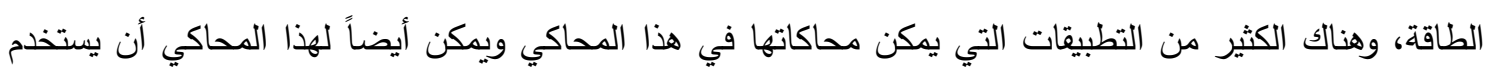

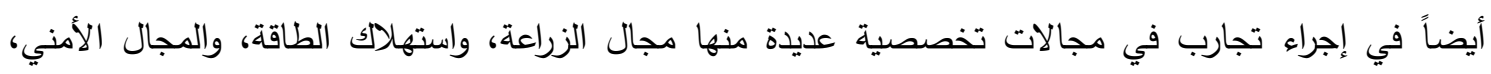


والاتصالات، والثبكات، والتقنيات الذكية، وتوجيه البيانات، وأنظمة المراقبة، وأنظمة مراقبة توزيع الأحمال على الخوادم وغيرها الكثير .

\section{Experiments Design}

6. تصميم التجارب

في هذا البحث، صُممت مجموعة كبيرة من التجارب لغرض تتفيذها وتحليل نتائجها؛ وتتميز تجارب هذا البحث بالتتوع في الإعدادات والخيارات التي تضفي على البحث طابعاً عملياً مميزاً وتتريه بالنتائج وتعززها من ون الناحية العلمية. وغاية هذا البحث هي تقصي تأثير نماذج الحركة على استهلاك مصادر الثبكة وتحليلها في المدن

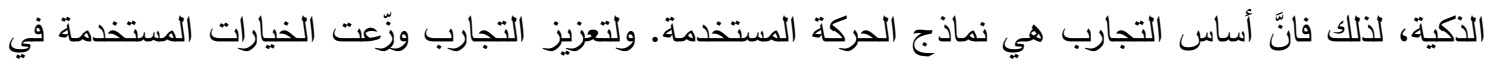
كل تجربة لتثمل خيارات متتوعة بتوزيع العقد وأيضاً خوارزميات متتوعة لأغراض نقل البيانات بين العقد. إن عدد

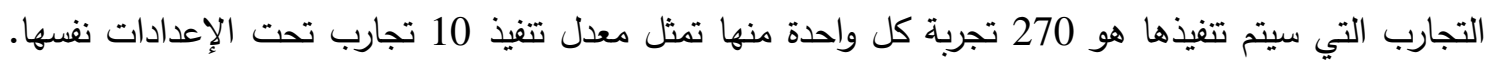

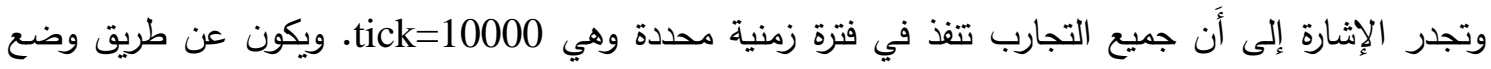

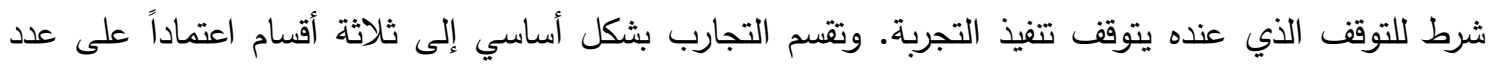

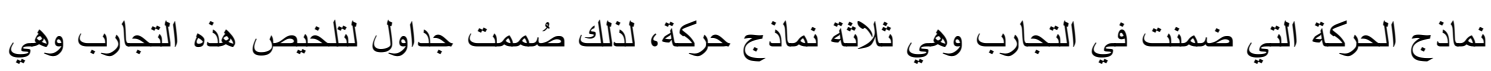
على النحو الآتي:

• المجموعة الأولى: تتكون من 90 تجربة تبدأ بالترميز EXP.01 إلى EXP.90. يتم في هذه المجموعة

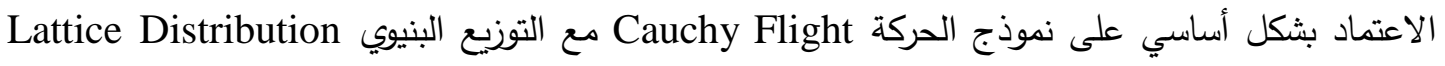

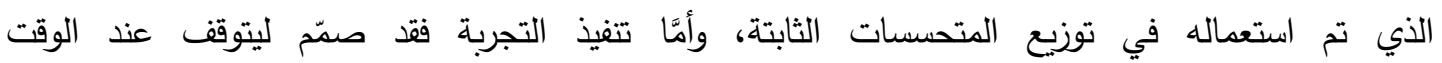

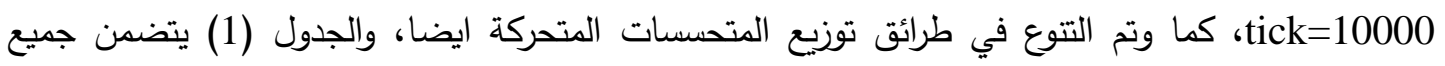
الإعدادات الأخرى للمجموعة الأولى من التجارب ويظهر من الجدول أنّ هناك تتوعاً في عدد العقد المتحركة

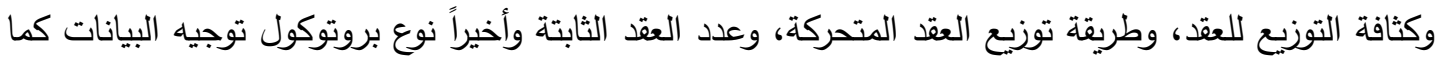

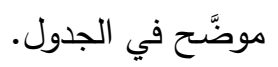

الجدول (1) المجموعة الأولى من التجارب

\begin{tabular}{|c|c|c|c|c|c|c|}
\hline \# & EXP. N. & Density & \# of mobile sensor & $\begin{array}{c}\text { mobile } \\
\text { sensor } \\
\text { distribution }\end{array}$ & $\begin{array}{c}\text { \# of } \\
\text { static } \\
\text { sensor }\end{array}$ & Routing Protocol \\
\hline 1 & $\begin{array}{l}\text { EXP. } \\
01-15\end{array}$ & $\begin{array}{c}1.96,2.94 \\
3.92,4.9,5.88\end{array}$ & $100,200,300,400,500$ & Power-law & 100 & $\begin{array}{c}\text { Epidemic, Spray \& Wait, } \\
\text { Probabilistic Flooding }\end{array}$ \\
\hline 2 & $\begin{array}{l}\text { EXP. } \\
16-30\end{array}$ & $\begin{array}{c}1.46,2.44 \\
3.42,4.4,5.38\end{array}$ & $100,200,300,400,500$ & Power-law & 50 & $\begin{array}{c}\text { Epidemic, Spray \& Wait, } \\
\text { Probabilistic Flooding }\end{array}$ \\
\hline 3 & $\begin{array}{l}\text { EXP. } \\
31-45\end{array}$ & $\begin{array}{c}1.96,2.94 \\
3.92,4.9,5.88\end{array}$ & $100,200,300,400,500$ & Uniform & 100 & $\begin{array}{c}\text { Epidemic, Spray \& Wait, } \\
\text { Probabilistic Flooding }\end{array}$ \\
\hline 4 & $\begin{array}{l}\text { EXP. } \\
46-60\end{array}$ & $\begin{array}{c}1.46,2.44 \\
3.42,4.4,5.38\end{array}$ & $100,200,300,400,500$ & Uniform & 50 & $\begin{array}{c}\text { Epidemic, Spray \& Wait, } \\
\text { Probabilistic Flooding }\end{array}$ \\
\hline 5 & $\begin{array}{l}\text { EXP. } \\
61-75\end{array}$ & $\begin{array}{c}1.96,2.94 \\
3.92,4.9,5.88\end{array}$ & $100,200,300,400,500$ & Normal & 100 & $\begin{array}{l}\text { Epidemic, Spray \& Wait, } \\
\text { Probabilistic Flooding }\end{array}$ \\
\hline 6 & $\begin{array}{l}\text { EXP. } \\
76-90\end{array}$ & $\begin{array}{c}1.46,2.44 \\
3.42,4.4,5.38\end{array}$ & $100,200,300,400,500$ & Normal & 50 & $\begin{array}{l}\text { Epidemic, Spray \& Wait, } \\
\text { Probabilistic Flooding }\end{array}$ \\
\hline
\end{tabular}


• المجموعة الثانية: تتكون من 90 تجربة تبدأ بالترميز EXP.91 إلى 180.

بشكل أساسي على نموذج الحركة Correlated Directions مع التوزيع البنيوي Lattice Distribution

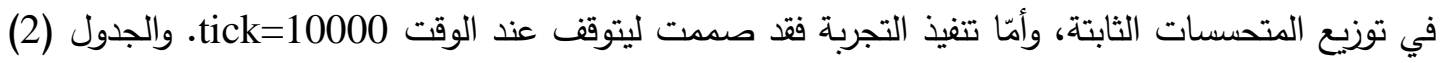

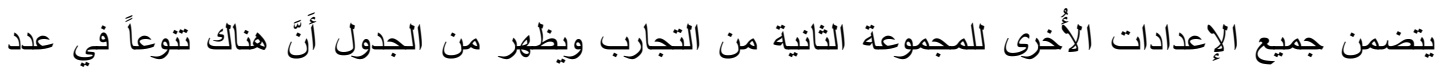

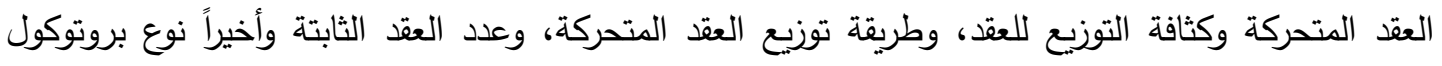

$$
\text { توجيه البيانات وكما موضح في الجدول. }
$$

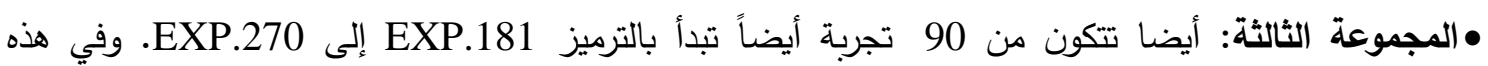
المجموعة اعتُمد بشكل أساسي على نموذج الحركة Human Mobility Model وهو نموذج يحاكي حركة الإنسان. وقد استعمل التوزيع البنيوي Lattice Distribution في توزيع المتحسات الثابتة. وأمَّا تتفيذ التجربة فقد صدّم ليتوقف بالوقت tick=10000. والجدول (3) يتضمن جميع الإعدادات الأخرى للمجموعة الأولى من

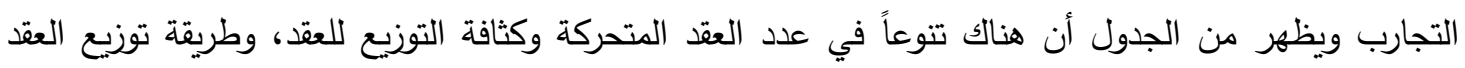
المتحركة، وعدد العقد الثابتة وأخيراً نوع بروتوكول توجيه البيانات كما موضح فئ في الجدول.

الجدول (2) المجموعة الثانية من التجارب

\begin{tabular}{|c|c|c|c|c|c|c|}
\hline \# & $\begin{array}{l}\text { EXP. } \\
\text { NO. }\end{array}$ & Density & No. of mobile sensor & $\begin{array}{c}\text { mobile } \\
\text { sensor } \\
\text { distribution }\end{array}$ & $\begin{array}{l}\text { No. of } \\
\text { static } \\
\text { sensor }\end{array}$ & Routing Protocol \\
\hline 1 & $\begin{array}{l}\text { EXP. } \\
91-105\end{array}$ & $\begin{array}{c}1.96,2.94 \\
3.92,4.9,5.88\end{array}$ & $100,200,300,400,500$ & Power-law & 100 & $\begin{array}{c}\text { Epidemic, Spray \& } \\
\text { Wait, Probabilistic } \\
\text { Flooding }\end{array}$ \\
\hline 2 & $\begin{array}{l}\text { EXP. } \\
106-120\end{array}$ & $\begin{array}{c}1.46,2.44, \\
3.42,4.4,5.38\end{array}$ & $100,200,300,400,500$ & Power-law & 50 & $\begin{array}{c}\text { Epidemic, Spray \& } \\
\text { Wait, Probabilistic } \\
\text { Flooding }\end{array}$ \\
\hline 3 & $\begin{array}{c}\text { EXP. } \\
121-135\end{array}$ & $\begin{array}{c}1.96,2.94 \\
3.92,4.9,5.88\end{array}$ & $100,200,300,400,500$ & Uniform & 100 & $\begin{array}{c}\text { Epidemic, Spray \& } \\
\text { Wait, Probabilistic } \\
\text { Flooding }\end{array}$ \\
\hline 4 & $\begin{array}{l}\text { EXP. } \\
136-150\end{array}$ & $\begin{array}{c}1.46,2.44 \\
3.42,4.4,5.38\end{array}$ & $100,200,300,400,500$ & Uniform & 50 & $\begin{array}{c}\text { Epidemic, Spray \& } \\
\text { Wait, Probabilistic } \\
\text { Flooding }\end{array}$ \\
\hline 5 & $\begin{array}{c}\text { EXP. } \\
151-165\end{array}$ & $\begin{array}{c}1.96,2.94 \\
3.92,4.9,5.88\end{array}$ & $100,200,300,400,500$ & Normal & 100 & $\begin{array}{c}\text { Epidemic, Spray \& } \\
\text { Wait, Probabilistic } \\
\text { Flooding }\end{array}$ \\
\hline 6 & $\begin{array}{l}\text { EXP. } \\
166-180\end{array}$ & $\begin{array}{c}1.46,2.44 \\
3.42,4.4,5.38\end{array}$ & $100,200,300,400,500$ & Normal & 50 & $\begin{array}{c}\text { Epidemic, Spray \& } \\
\text { Wait, Probabilistic } \\
\text { Flooding }\end{array}$ \\
\hline
\end{tabular}


الجدول (3) المجموعة الثالثة من التجارب

\begin{tabular}{|c|c|c|c|c|c|c|}
\hline \# & $\begin{array}{l}\text { EXP. } \\
\text { NO. }\end{array}$ & Density & No. of mobile sensor & $\begin{array}{c}\text { mobile } \\
\text { sensor } \\
\text { distribution }\end{array}$ & $\begin{array}{l}\text { No. of } \\
\text { static } \\
\text { sensor }\end{array}$ & Routing Protocol \\
\hline 1 & $\begin{array}{c}\text { EXP. } \\
181-195\end{array}$ & $\begin{array}{c}1.96,2.94 \\
3.92,4.9,5.88\end{array}$ & $100,200,300,400,500$ & Power-law & 100 & $\begin{array}{c}\text { Epidemic, Spray \& } \\
\text { Wait, Probabilistic } \\
\text { Flooding }\end{array}$ \\
\hline 2 & $\begin{array}{c}\text { EXP. } \\
196-210\end{array}$ & $\begin{array}{c}1.46,2.44 \\
3.42,4.4,5.38\end{array}$ & $100,200,300,400,500$ & Power-law & 50 & $\begin{array}{c}\text { Epidemic, Spray \& } \\
\text { Wait, Probabilistic } \\
\text { Flooding }\end{array}$ \\
\hline 3 & $\begin{array}{c}\text { EXP. } \\
211-235\end{array}$ & $\begin{array}{c}1.96,2.94 \\
3.92,4.9,5.88\end{array}$ & $100,200,300,400,500$ & Uniform & 100 & $\begin{array}{c}\text { Epidemic, Spray \& } \\
\text { Wait, Probabilistic } \\
\text { Flooding }\end{array}$ \\
\hline 4 & $\begin{array}{c}\text { EXP. } \\
236-250\end{array}$ & $\begin{array}{c}1.46,2.44 \\
3.42,4.4,5.38\end{array}$ & $100,200,300,400,500$ & Uniform & 50 & $\begin{array}{c}\text { Epidemic, Spray \& } \\
\text { Wait, Probabilistic } \\
\text { Flooding }\end{array}$ \\
\hline 5 & $\begin{array}{c}\text { EXP. } \\
251-265\end{array}$ & $\begin{array}{c}1.96,2.94 \\
3.92,4.9,5.88\end{array}$ & $100,200,300,400,500$ & Normal & 100 & $\begin{array}{c}\text { Epidemic, Spray \& } \\
\text { Wait, Probabilistic } \\
\text { Flooding }\end{array}$ \\
\hline 6 & $\begin{array}{c}\text { EXP. } \\
266-270\end{array}$ & $\begin{array}{c}1.46,2.44 \\
3.42,4.4,5.38\end{array}$ & $100,200,300,400,500$ & Normal & 50 & $\begin{array}{c}\text { Epidemic, Spray \& } \\
\text { Wait, Probabilistic } \\
\text { Flooding }\end{array}$ \\
\hline
\end{tabular}

سنحلّل النتائج التي حصلنا عليها من التجارب التي صُمدت وشُرحت في القسم السابق، وطريقة عرض النتائج وتحليلها في هذا البحث تستند بالدرجة الأساس على نموذج الحركة المستخدم، كما تعتمد بالدرجة الثانية على طريقة توجيه البيانات في الثبكة.

\section{Network Resources Consumption}

لغرض التقليل من الهدر للموارد والجهد في الددن الذكية، يجب ان يتم الحفاظ على الاداء العام لاستهلاك

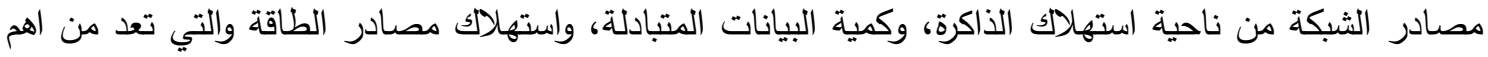
التحديات التي يواجهها مطورو المدن الذكية، والتي سيتم توضيحها الان:

\section{Amount of Data Exchanged}

1-1-7 - 2 1-1 كمية البيانات المتبادلة

يعدّ هذا الجزء من أَهم أَجزاء البحث، لأَنَّه يعكس سلوك استهلاك مصادر الثبكة. عملياً، إن كمية البيانات

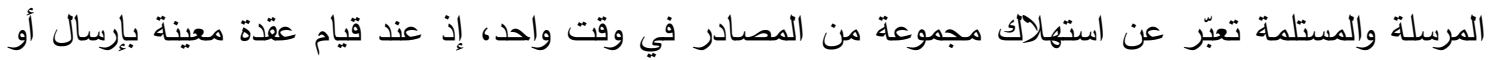
استلام رسالة معينة سوف يتم استهلاك مصادر الطاقة والذاكرة لتلك العقدة. فضلا عن استهلاك مسار لنقل البيانات؛ لذلك من الضروري فهم وإدراك عمليات تبادل البيانات بين عقد الثبكة. إذ ان الثكل (1) يعكس سلوك نماذج الحركة المستخدمة Correlated model و Cauchy flight و باستخدام توزيع Power-Law للعقد المتحركة مع طريقة من نوع Epidemic لنقل البيانات، وفي هذا الثكل يمثل المحور عدد العقد المستخدمة وقد استُعمل عدد متغير؛ لغرض ملاحظة التغييرات التي تحصل في حال زيادة كمية 
العقد. فقد استُعمل(100،200،300،400، ب فيتضمن كمية البيانات المتبادلة التي تمثل بدورها عدد الرسائل المتبادلة، ونلاحظ في هذا الثكل إن كمية البيانات المتبادلة باستخدام نموذج حركة الإنسان تصل لأقل حد لها مقارنة بالأنموذجين الآخرين. ونتيجة هذا التفوق لنموذج حركة الإنسان

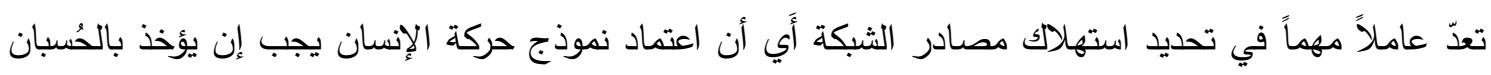
من قبل مطوري المدن الذكية.

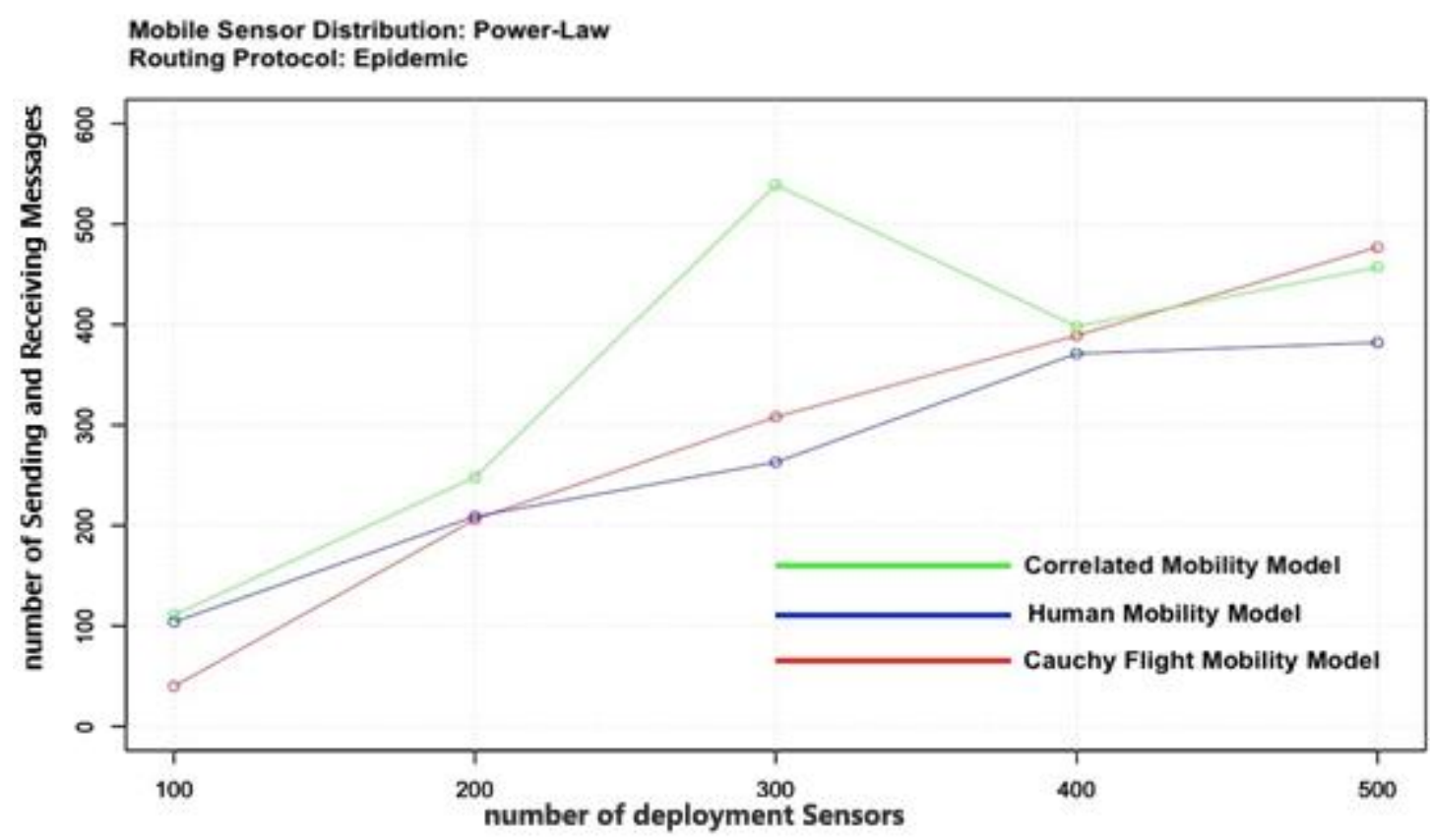

الثكل (1) كمية البيانات المستهلكة عند استخدام توزيع Power-Law مع طريقة Epidemicفي توجيه البيانات عند

$$
\text { توظيف نماذج الحركات الثلاث. }
$$

ولغرض تفحص النتائج بثكل أدق، تم التقصي عن التغيرات التي حصلت في تتفيذ التجارب، ولهذا السبب

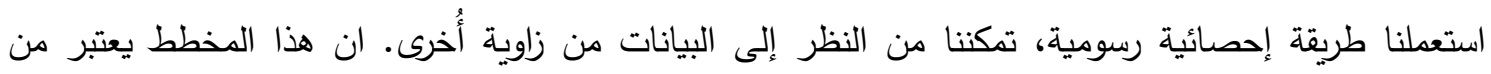
الطرق الاحصائية للكثف عن مدى استقرارية سلوك معين وفي هذا البحث تم استخدامه لبيان استقرارية النماذج إنج المستخدمة وبالتالي التعرف على مدى وثوقية اداء النماذج. والثكل (2) يوضح التغيرات التي تحصل في كمية فئل

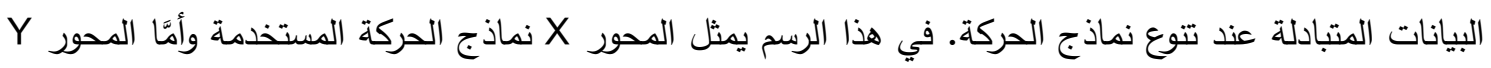
فيمثل كمية البيانات المتبادلة. ونلاحظ أنّ استقرارية نموذج حركة الإنسان يفوق بمقدار قلئليل استقرارية النموذجين الآخرين. بمعنى آخر، إن تضمين نموذج حركة الإنسان يعطي نتائج تفوق في استقراريتها النماذج الأخرى 


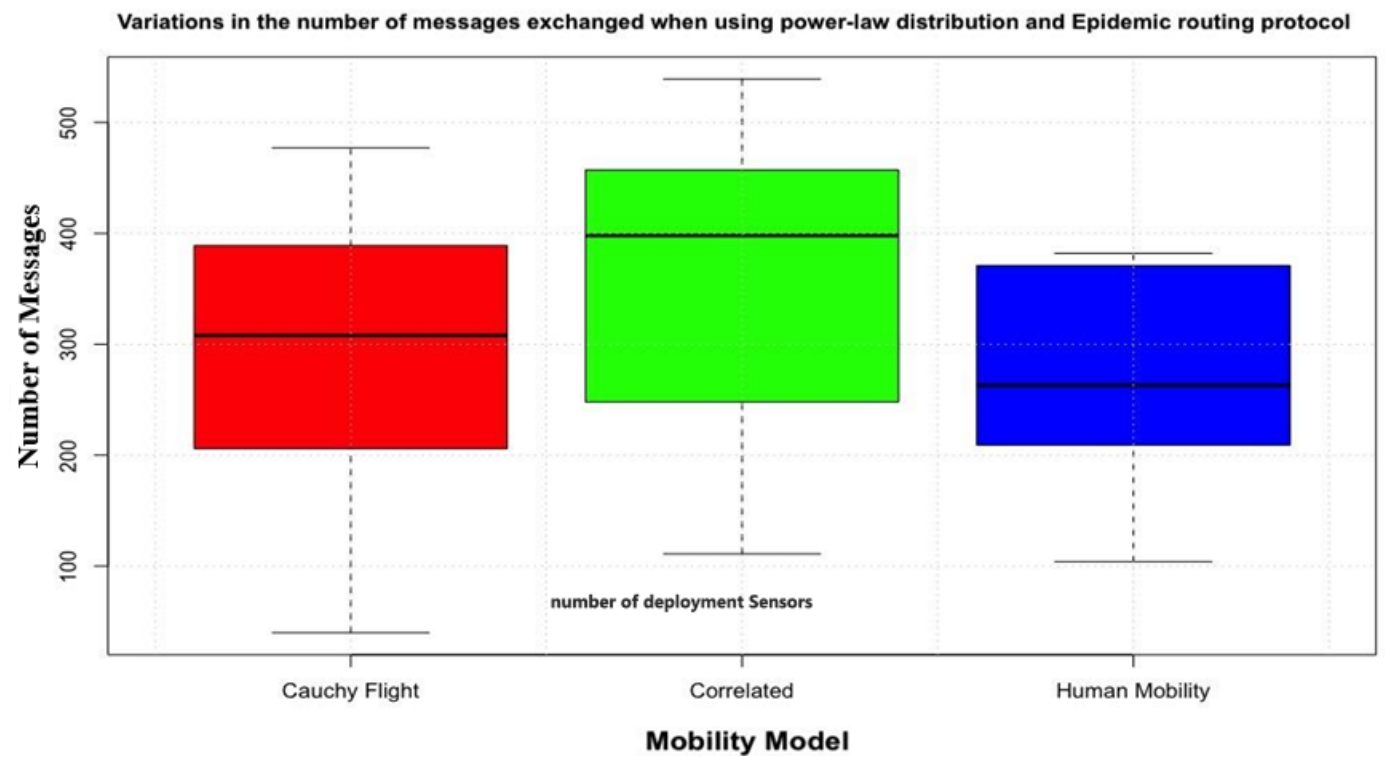

الثكل (2) قياس استقرارية تبادل البيانات في نماذج الحركة المستخدمة التي اعتمد طريقة Epidemic في نقل البيانات

إن جميع ما ذكر من تحليل هو خاص بالتجارب التي أُجريت باستخدام طريقة Epidemic لنقل البيانات. والثكل (3) يعكس تنفيذ التجارب باستخدام خوارزمية Spray\&Wait لنقل البيانات في الثبكة وفي هذا الثكل،

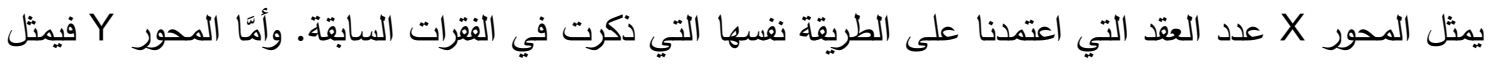
كمية البيانات المتبادلة، ومن المشير للاهتمام في هذا الثكل هو تفوق واضح لنموذج الحركة Flight Cauchy

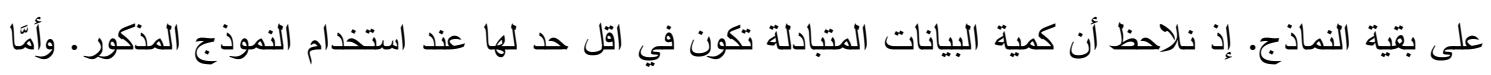

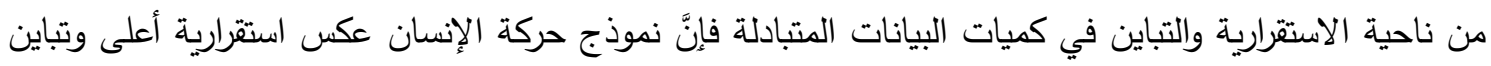

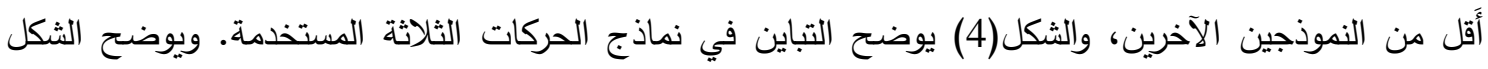
أيضاً ظهور قيمة متطرفة Outlier في نموذج حركة الإنسان التي من بعد تدقيقنا للبيانات تبيّن أنّها نتيجة متطرفة وبنة لأحدى التجارب ولم تتكرر إلاًّ لمرة واحدة؛ ولأجل هكذا حالات نفذت كل تجربة عشرة مرات واعتماد معدلها كنتيجة

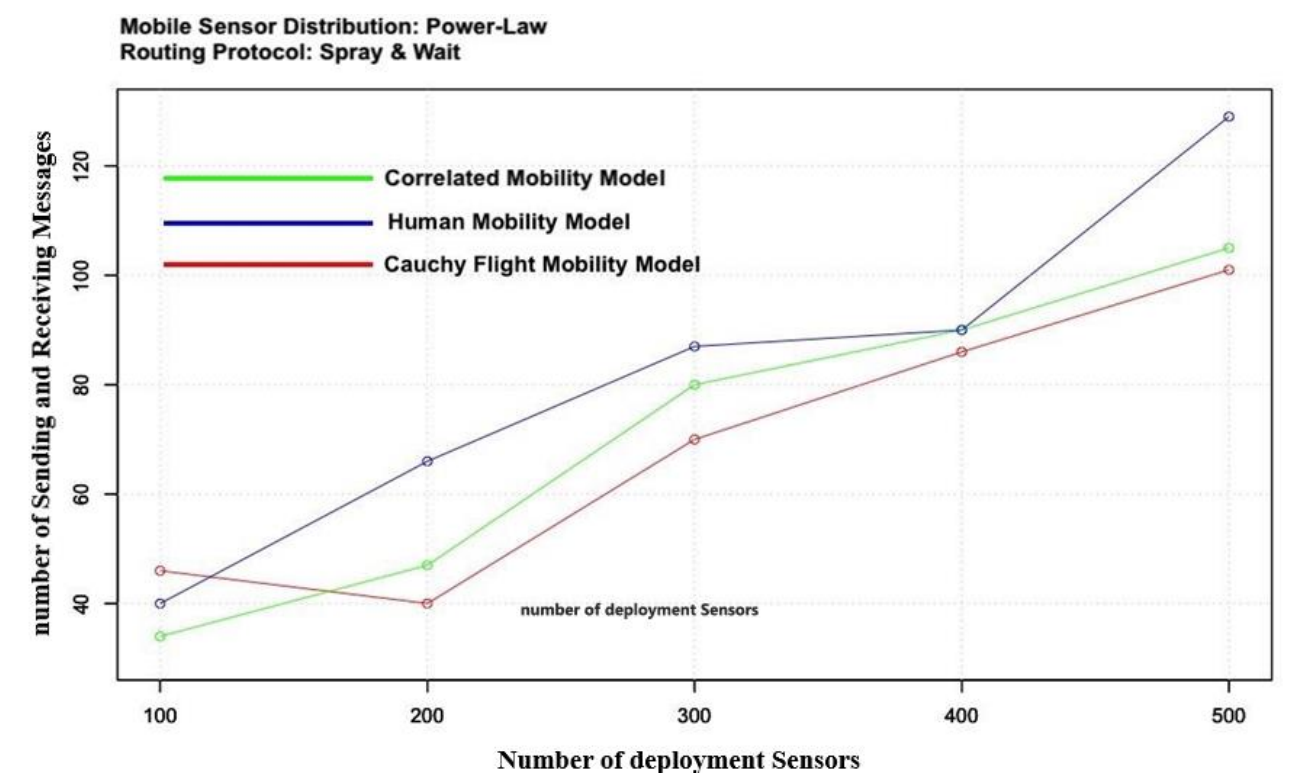

الثكل (3) كمية البيانات المستهلكة عند استخدام توزيع Power-Law مع خوارزمية Spray \& Wait في توجيه البيانات عند توظيف نماذج الحركات الثلاث. 


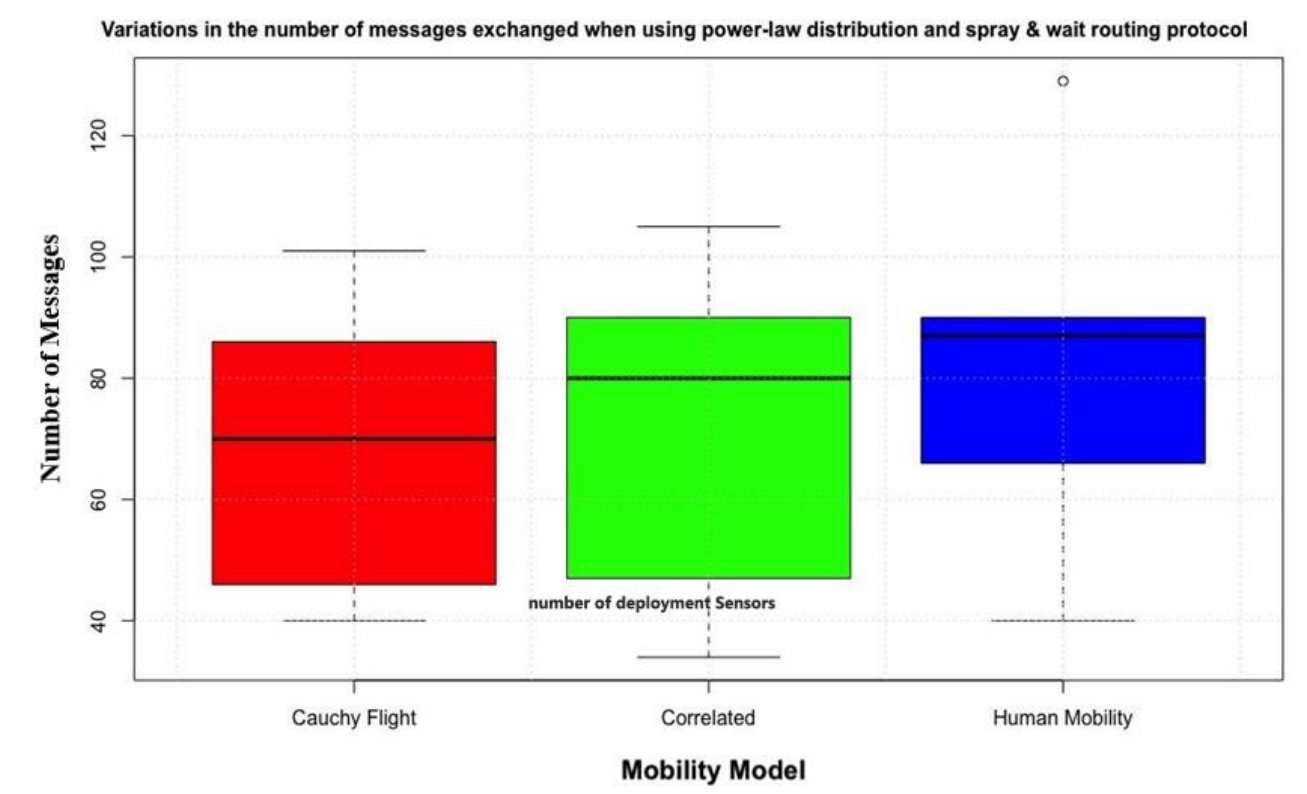

الثكل (4) قياس استقرارية تبادل البيانات في نماذج الحركة المستخدمة التي اعتمدت طريقة Spray \& Wait في نقل

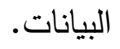

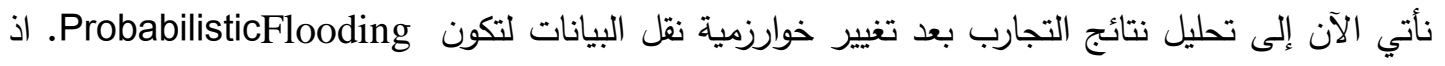

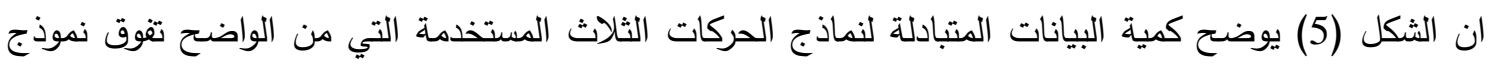

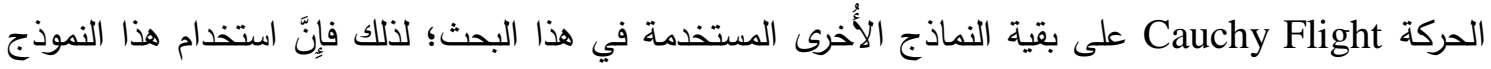
من الحركة يعدّ فعالاً باستخدام خوارزمية Probabilistic Flooding و و Spray \& Wait في نقل البيانات وأنّه يحافظ على إستهلاك مصادر الشبكة.

Mobile Sensor Distribution: Power-Law

Routing Protocol: Probabilistic Flooding

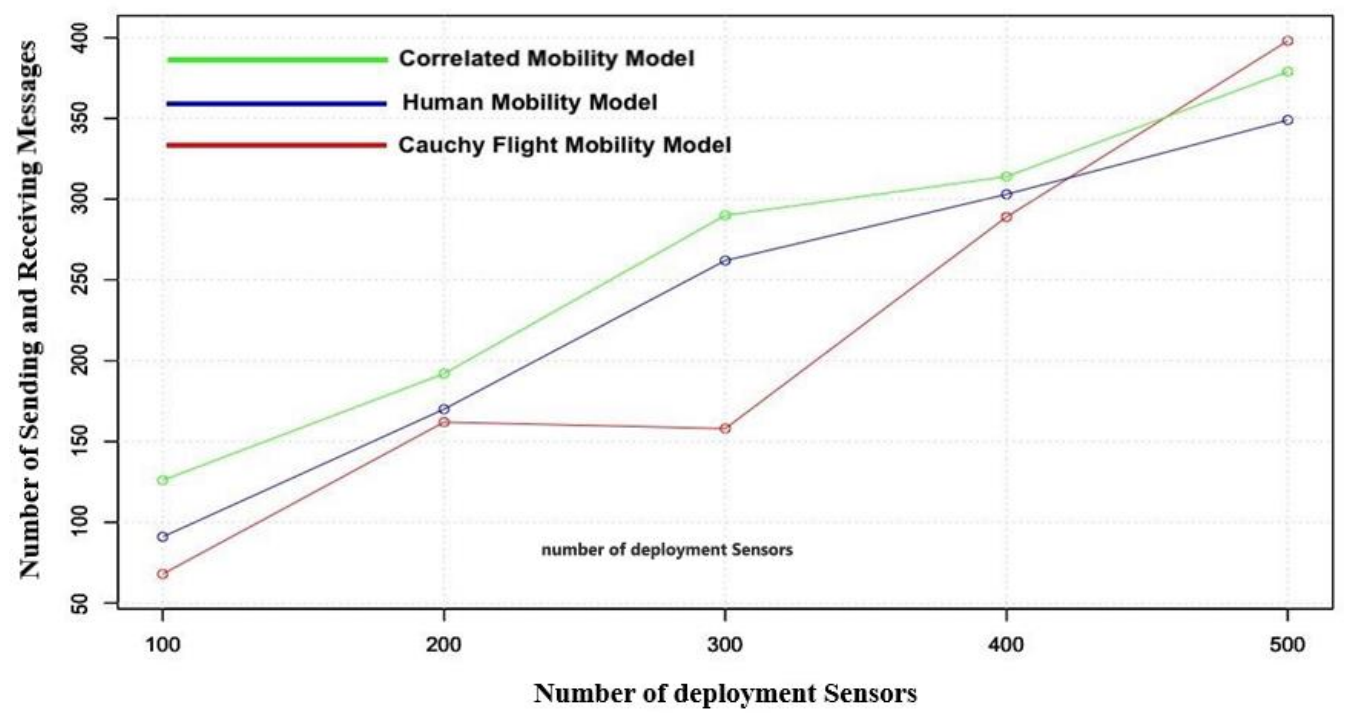

الثكل (5) كمية البيانات المستهكة عند استخدام توزيع Power-Law مع خوارزمية Probabilistic Flooding في توجيه البيانات عند توظيف نماذج الحركات الثلاث. 
من ناحية أُخرى، هناك نقطة سلبية في أداء نموذج الحركة Cauchy Flight وهي عدم استقرارية النموذج وتباين نتائجه في مختلف التجارب. والثكل (6) يصور أداء نماذج الحركات الثلاث الذي من الواضح فيه التذبذب نداج في استقرارية وتباين كمية البيانات المتبادلة. Variations in the number of messages exchanged when using power-law distribution and probabilistic flooding routing protocol

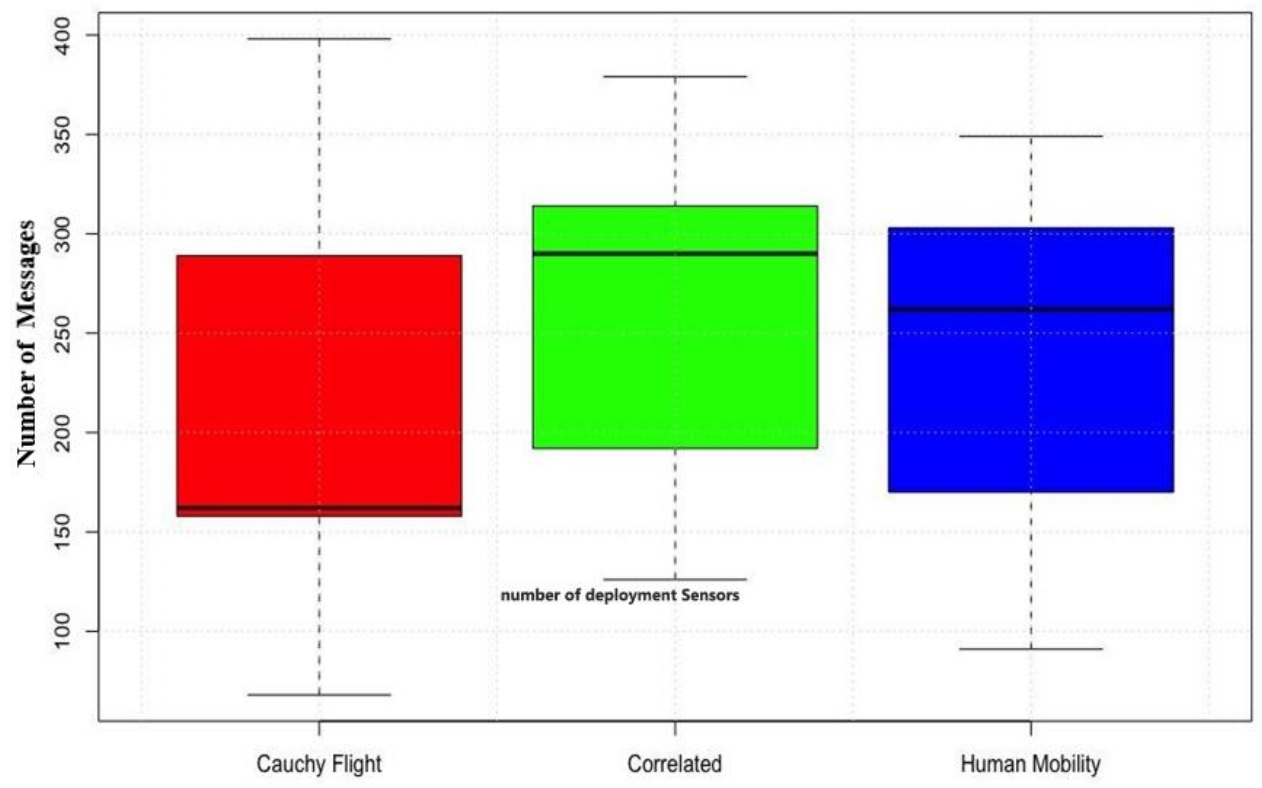

Mobility Model

الثكل (6) قياس استقرارية تبادل البيانات في نماذج الحركة المستخدمة التي اعتمد طريقة Probabilistic Flooding في نقل البيانات.

\section{Power Resources Consumption}

2-1-7 استهلاك مصادر الطاقة

يعذّ تقليل استهلاك الطاقة من أهم التحديات التي يواجهها مطورو المدن الذكية، وكذلك شبكات المتحسات اللاسلكية لأََّّ هذه المتحسسات متحركة ولا تمتلك مصدر مستمر للطاقة؛ لذلك يُعتمَد على بطارية هذه المتحسسات فقط مصدراً رئيساً للطاقة وفي الحقيقة هناك الكثير من العوامل التي تؤثر على استهلاك الطاقة ومن أهمها هي نئي كمية البيانات المتبادلة التي شُرحت في الفقرات أعلاه. في هذا البحث، افترضنا أن جميع العقد المتحركة في المدينة

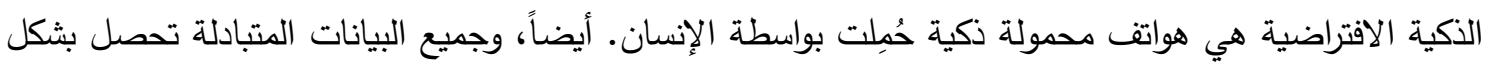
رئيس عن طريق تقنية Bluetooth وأَنَنّا اعتمدنا الأولى في هذا البحث. إن كل عملية إرسال أو استلام

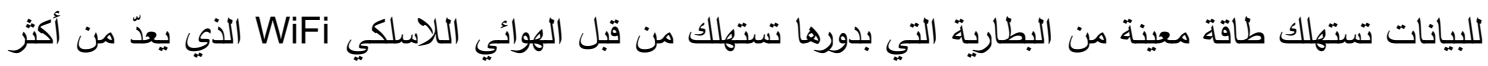

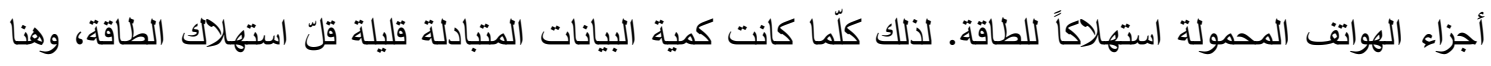
يجب الإثارة إلى أن تقليل كمية البيانات المتبادلة لا يجب أن يكون على حساب الهدف المرجو من تصميم الثبكة. والثكل (7) يوضّح التباين في أعداد العقد التي استثعرت الحدث المنتشر في الثبكة باستخدام نماذج لهن الحركات الثلاث المعتمدة في هذا البحث، واستُعملت طرائق عديدة لنقل البيانات (Spray \& Wait،Epidemic

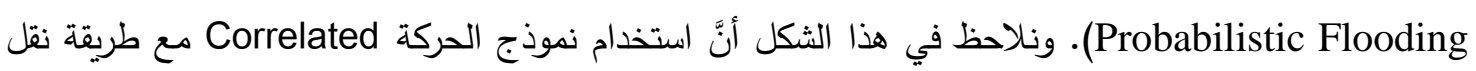
من نوع Probabilistic Flooding قد فاق أدائه النماذج الأخرى من ناحية عدد العقد التي استلمت البيانات (رسالة أو حدث). إن جميع النتائج التي حصلنا عليها باستخدام طريقة النقل Epidemic لا يُعتمد عليها لأنَّ هذه النّان الطريقة تُتخدم فقط لأغراض المقارنة والهدف دائماً هو الحصول على أداء قريب من أدائها. 


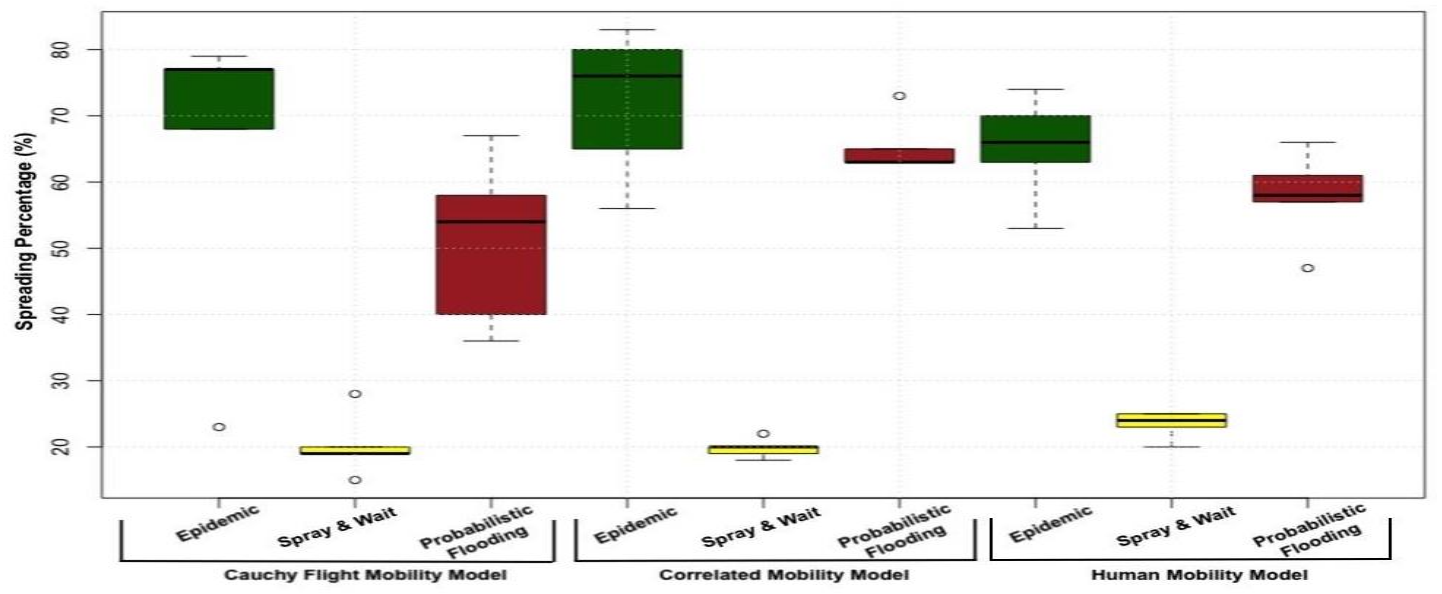

الثكل (7) النسبة المئوية لعدد العقد التي غُطِيت بالبيانات في جميع نماذج الحركة المستخدمة في هذا البحث وجميع

خوارزميات نقل البيانات المستخدمة أيضاً.

\section{Memory Consumption استهلاك الذاكرة}

يعد استهلاك الذاكرة من المعايير المهمة في قياس الأداء العام للشبكة، ولتوضيح هذه المسألة نفترض وجود هاتف محمول الذي يعمل على تقنية WiFi في نقل البيانات. في هذه الحالة، حينما يكون حامل هذا الجهاز في منطقة مزدحمة كأن تكون سوق أو مول تجاري فإنَّ عدد الهواتف المحمولة التي تكون في مدى هذا الهاتف كثيرة جداً، وفي هذه الحالة على افتراض وجود مدينة ذكية، إذا ما استُلمت رسائل من الأجهزة المحيطة فإنَّ ذاكرة هذا الجهاز ستكون ممتلئة في أسابيع معينة؛ لذلك يجب إدارة الذاكرة وتحديد ما سوف يخزن بها من بيانات والميل إلى دانى عدم خزن بيانات غير قيمة لذلك الجهاز، والثكل (8) يوضح أداء نماذج الحركات الثلاث المستخدمة وطرائق النقل الثلاث أيضاً. ونلاحظ من هذا الثكل أنَّ خوارزمية توجيه البيانات من نوع Spray \& Wait باستخدام

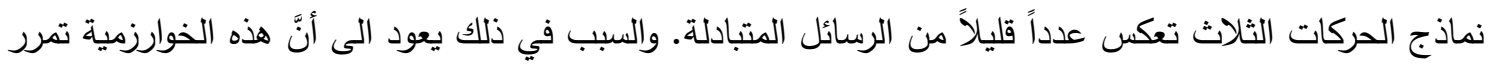
البيانات أو الرسائل بطريقة محددة جداً، وهذا ما يؤدي بالتالي إلى تقليل استهلاك مصادر الثبكة من ناحية الذاكرة ومن نواحي أخرى مثل استهلاك الطاقة.

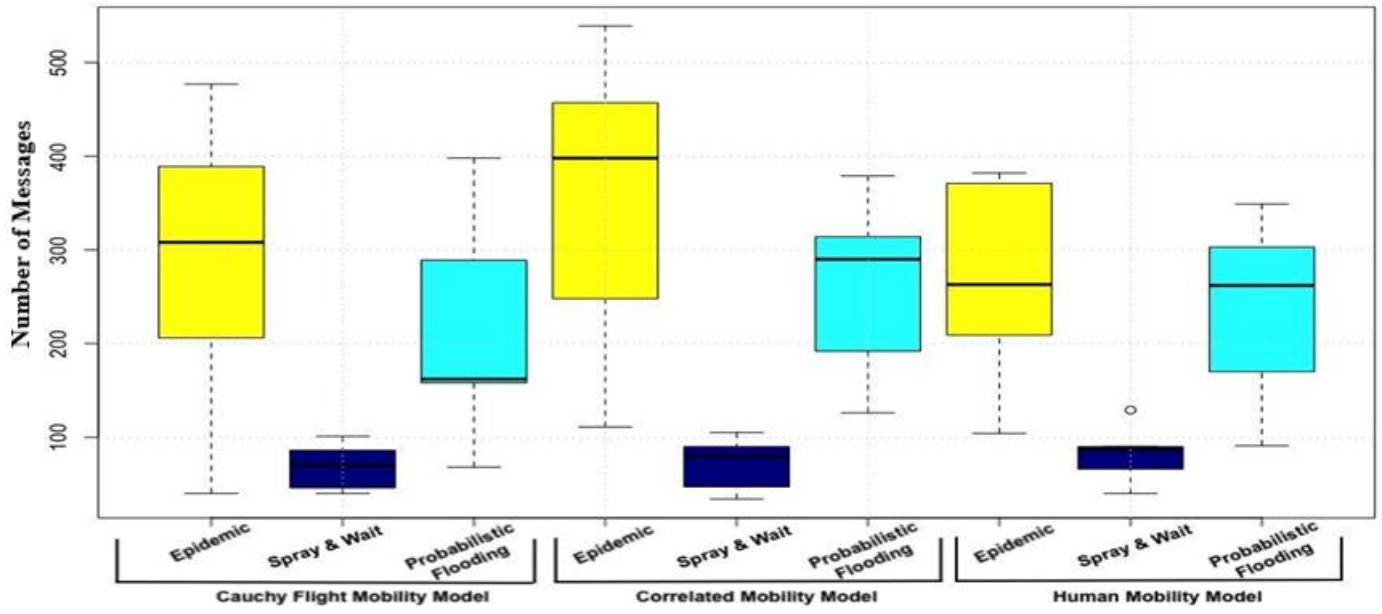

الثكل (8) عدد الرسائل المتبادلة باستخدام جميع نماذج الحركة في هذا البحث وجميع خوارزميات نقل البيانات المستخدمة أيضا. 


\section{Coverage Area}

2-7 - 2-7 مساحة التغطية

من القياسات التي أُجريت في هذا البحث هي المساحة التي غُطيت بالبيانات في الثبكة. إذ استُعملت نماذج

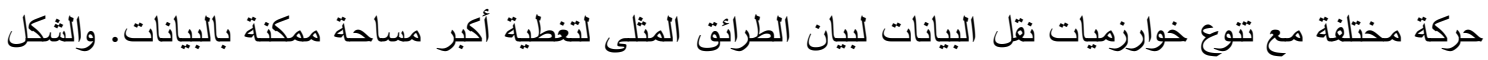
(9) يوضح تفوق واضح لنموذج الحركة Correlated مقارنة مع النموذجين الآخرين عند استخدام طريقة نقل

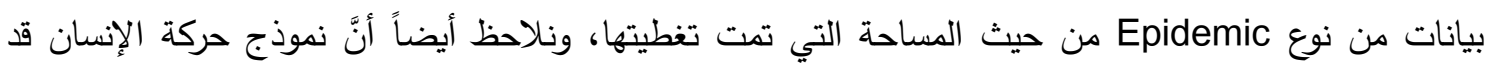
عكس اداءاً مقارباً جداً إلى النموذج المذكور آنفاً. فيما تخلف نموذج الحركة Cauchy Flight في أدائه بشكل واضحح.

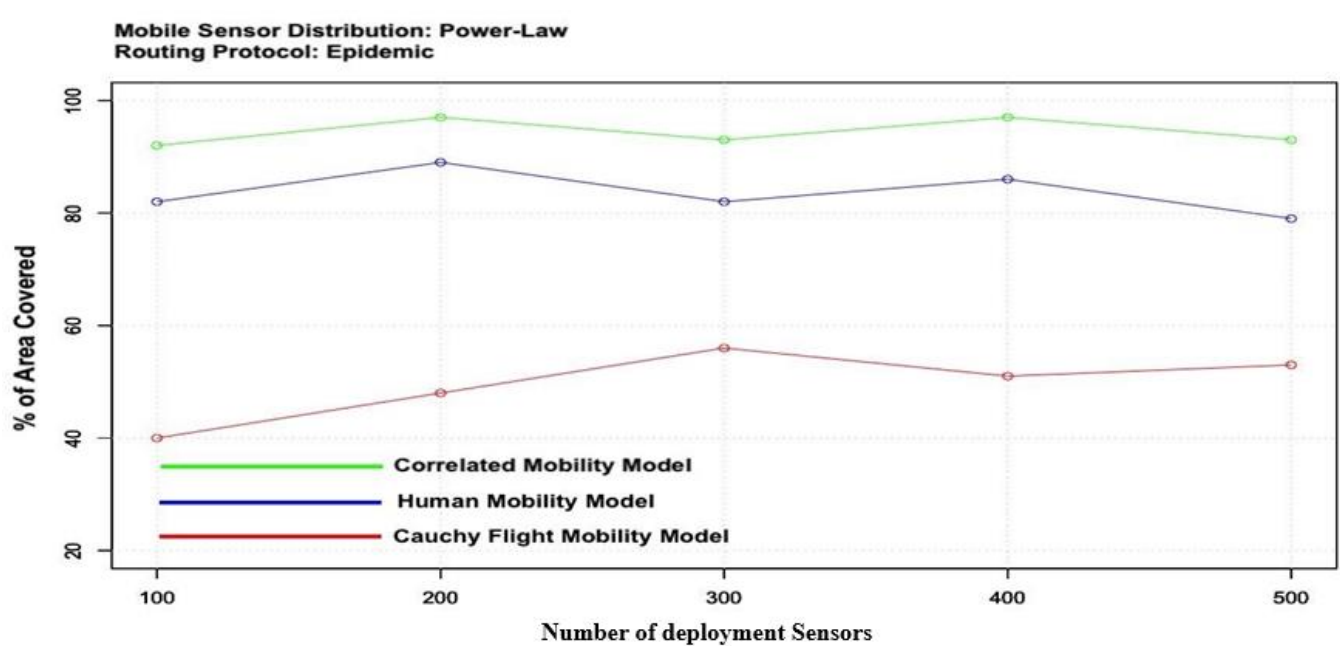

الثكل (9) المساحة التي غُطيت بالبيانات باستخدام توزيع Power-Law مع خوارزمية Epidemic في توجيه البيانات عند توظيف نماذج الحركات الثلاث.

أما الثكل (10) فإنَّه يعكس نتيجة مقاربة للثكل السابق باستخدام الإعدادات السابقة نفسها مع تغير طريقة

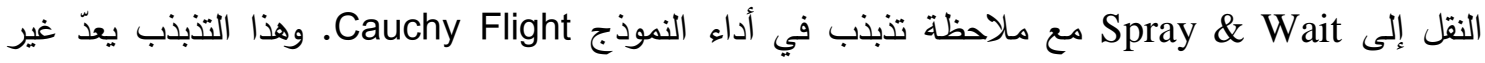
مرغوباً فيه لعدم استقراريته، ولا يمكن الاعتماد عليه في هذا النوع من الثبكات لأنَّ استقرارية نتائج تجربة معينة تعدّ من الأمور المهمة في التعبير عن مدى الاعتمادية عليها. Mobile Sensor Distribution: Power-Law Routing Protocol: Spray \& Wait

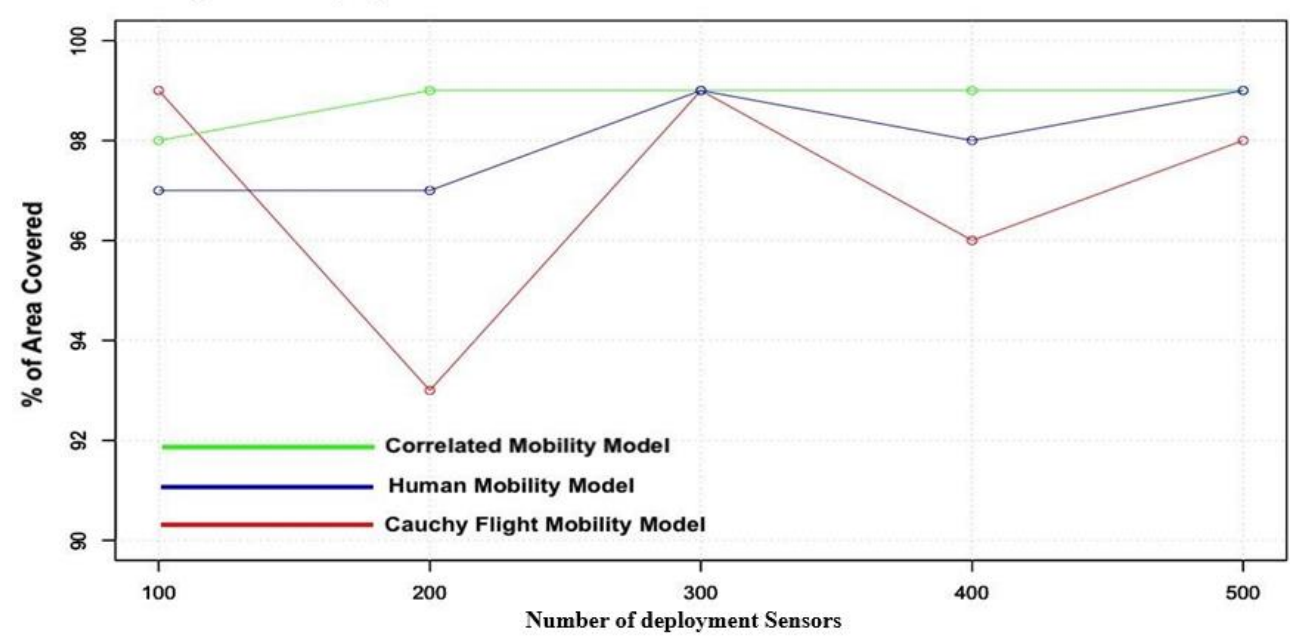

الثكل (10) المساحة التي غُطيت بالبيانات باستخدام توزيع Power-Law مع خوارزمية Spray\&Wait في توجيه البيانات عند توظيف نماذج الحركات الثلاث. 
في الثكل (11) غُيرّت طريقة نقل البيانات إلى Probabilistic Flooding الذي بدوره قد حسن من أداء نموذج الحركة Cauchy Flight ليتفوق على نموذج حركة الإنسان في المساحة التي غُطيت بالبيانات. وأمّا نموذج الحركة Correlated فقد بقي متفوقاً في أدائه عن بقية نماذج الحركة المستخدمة في هذا البحث. وتبيّن بالأشكال الثلاثة السابقة أن نموذج الحركة Correlated يعدّ من أفضل نماذج الحركة التي لها القابلية على تغطية أكبر مساحة ممكنة بالبيانات في الثبكة. وهذه النتيجة مستقرة حتى عند تغيير خوارزميات نقل وتوجيه البيانات. وتعدّ هذه النتيجة مثيرة للاهتمام بحيث إذا كان الهدف في الثبكة في وقت معين فمن الممكن الاعتماد على العقد التي تتبع في حركتها هذا النموذج في نشر البيانات إلى أكبر مساحات مدكنة في الثبكة في المدينة

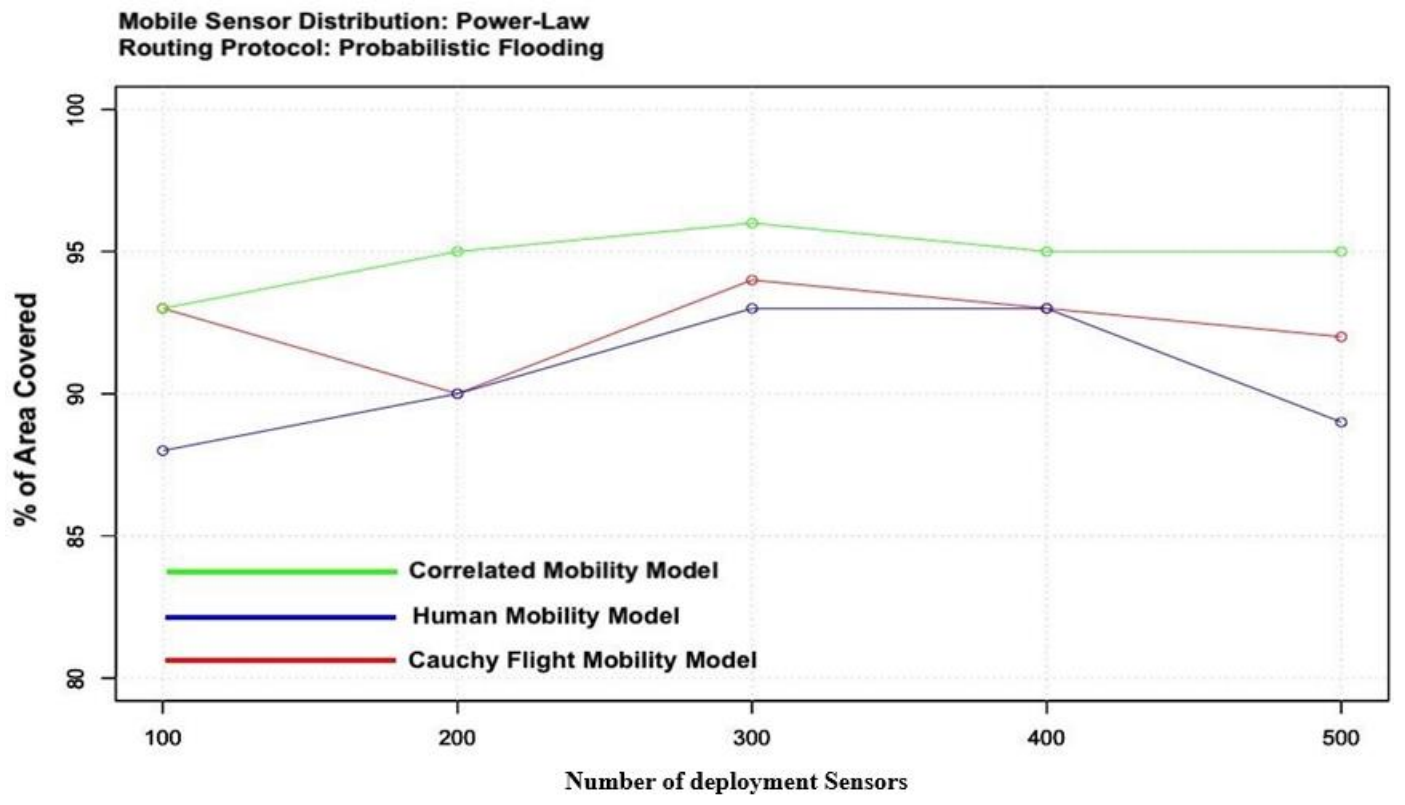

الثكل (11) المساحة التي غُطيت بالبيانات باستخدام توزيع Power-Law مع خوارزمية Probabilistic Flooding في توجيه البيانات عند توظيف نماذج الحركات الثلاث.

\section{8. المناقشة والإستنتاجات والأَعمال المستقبلية}

في هذا البحث قمنا بعمل محاكاة لمدينة ذكية تحمل مفهوم إنترنت الأشياء وتتضَّمن في تصميمها شبكة لاسلكية. وهذه الثبكة تتكون من عقد ثابتة ومتحركة. بحيث أن كل عقدة ثابتة تمثل متحسس أو مستثعر في مكان

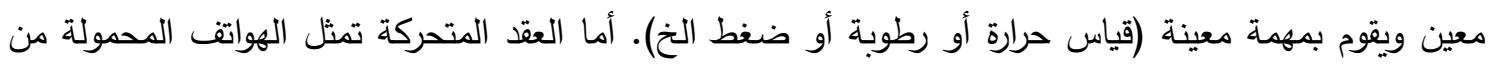

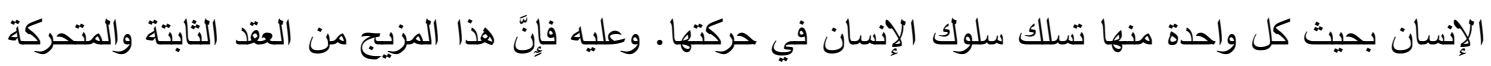
يخضع إلى عدة إعدادات التي تعكس في طبيعة تصميمها الكثير من المتغيرات. ومن أهم هذه المتغيرات هو نموذج

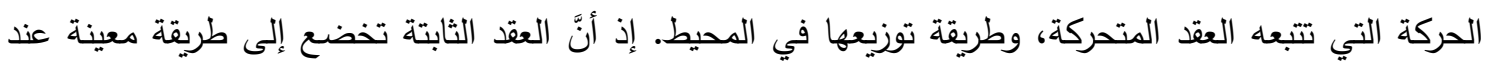
توزيعها. فضلاً عن ذلك، جميع العقد الثابتة والمتحركة يجب أن تستخدم طريقة أو خوارزمية معينة في تبادل البيانات فيما بينها.

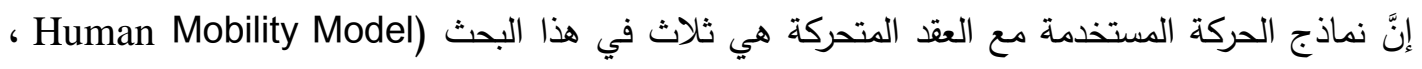
Power-Law) Correlated Model Probabilistic Spray\&Wait Epidemic)، Uniform 
Flooding). وقد صُممت 270 تجربة ونفذت في الوقت نفسه، وكل واحدة منها تمثل مزيج من المتغيرات المذكورة

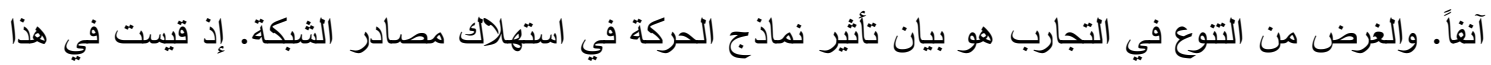
البحث أمور عديدة منها: كمية البيانات المتبادلة، واستهلاك مصادر الطاقة، واستهلاك الذاكرة. وأُجريت قياسات

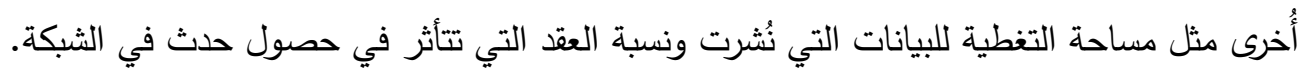
ويمكن تلخيص ما توصلنا إليه من نتائج في هذا البحث بالآتي: تعدّ نماذج الحركة التي استُعملت مع العقد المتحركة من أحد العوامل المهمة في تحديد استهلاك مصادر الثبكة. فضلاً عن تأثير نماذج الحركة في استهلاك مصادر الثبكة، فإنَّ خوارزميات وطرائق توجيه البيانات لها تأثير • يعدّ نموذج حركة الإنسان Human Mobility Model الذي يعبر بدقة عن سلوك الإنسان في الحركة من أكثر النماذج استقراراً من ناحية الأداء. على سبيل المثال، التباين الذي يحصل عندان عند تنفيذ التجارب في كمية

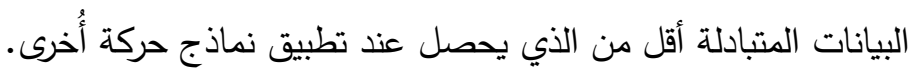

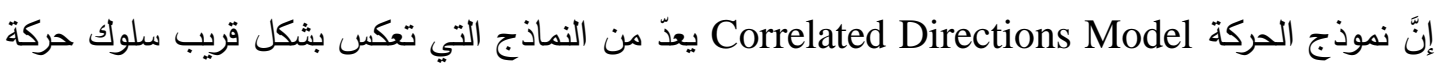
المركبات من النماذج التي تؤدي إلى تقليل استهلاك مصادر الثبكة ولكن في استقرارية أقل من نموذج حركة الإنسان. • استتاداً إلى النقطتين اعلاه من الممكن تطبيق نموذجي الحركة المذكورين في نموذج واحد والذي نعتقد أنه

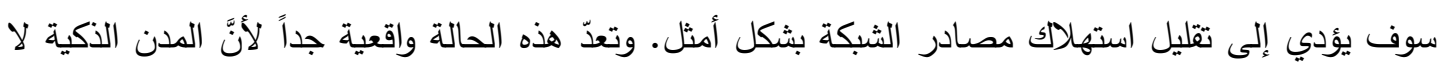
تضمّ أشخاصاً وإنَّا تضمّ مركبات أيضاً التي يمكن استغلالها على شكل حاملات للعقد المتحركة، ومن الممكن أن تساعد العقد المحمولة من الأشخاص أنفههم.

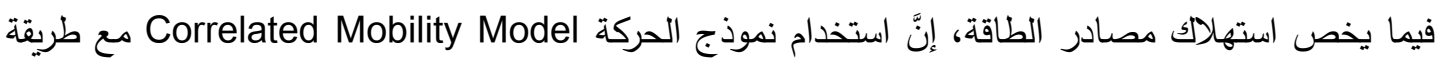
نقل من نوع Probabilistic Flooding قد فاق في أدائه النماذج الأخرى من ناحية عدد العقد التي استشعرت الأحداث في الثبكة. فيما يخص استهلاك ذواكر عقد الثبكة، إنَّ خوارزمية توجيه البيانات من نوع Spray\&Wait باستخدام نماذج

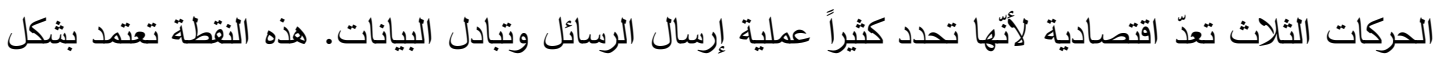
كبير على أهداف تصميم الثبكة، وعلى سبيل المثال إذا كان الحدث المراد نشره في الثبكة يعدّ إعلاناً أو تتبيهاً معيناً فإنَّ هذه الخوارزمية تكون غير فعالة. • يعدّ نموذج حركة الإنسان من النماذج التي لها القدرة على تغطية أكبر مساحة ممكنة في الثبكة بالبيانات. ويُعزى السبب في ذلك إلى كون إنَّ حركة الإنسان تكون غير مقيدة بمسارات معينة ومن الممكن للأشخاص التحرك باتجاهات ومسارات كثيرة ومختلفة. • أَثتبت التجارب أنَّ نموذج حركة الإنسان يعدّ من النماذج التي تتمتع باستقرارية في سلوكها وأدائها على الرغم

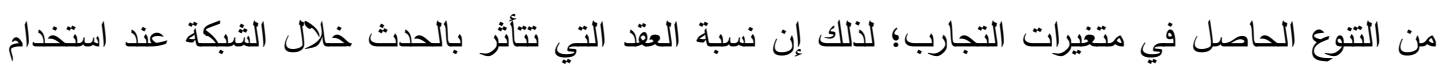
نموذج حركة الإنسان لا يعدّ أَمثل، ولكنَّه كفوء مقارنة مع بقية نماذج الحركة المستخدمة. • إنَّ قياس أداء تجربة معينة لا يعتمد فقط على النتائج التي حصلنا عليها من التجربة نفسها، ولكنَّهَ يعتمد أيضاً

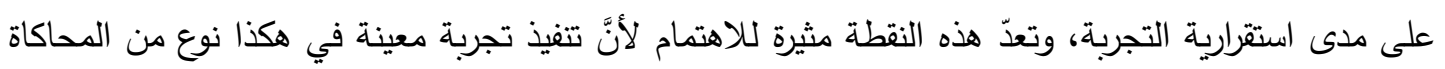


قد يعطي نتائج مختلفة في كل تتفيذ. لذلك قمنا في هذا البحث بتنفيذ كل تجربة عشر مرات والاعتماد على معدلها في تثبيت نتائج تجربة معينة. وأخيراً، من الأعمال المستقبلية التي يمكن إجراؤها في محاكاة المدن الذكية، وإنترنت الأثياء هو استخدام

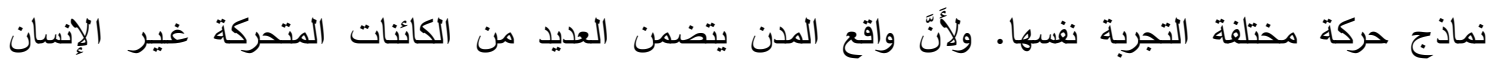

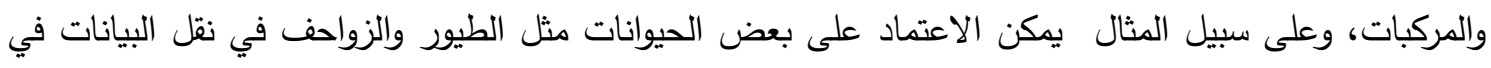

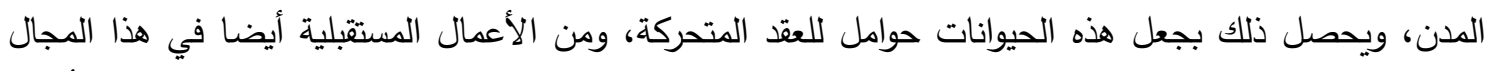

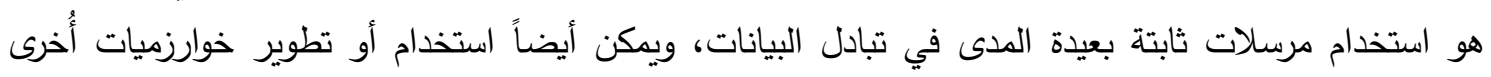

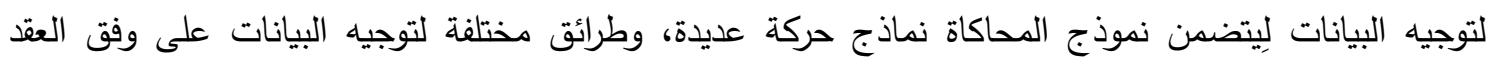

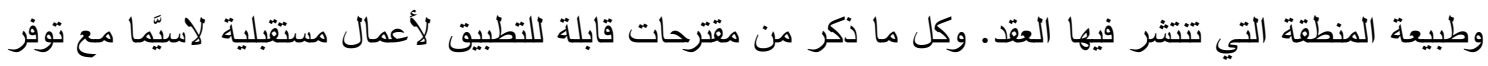
الأدوات البرمجية والتطبيقات التي تمكننا من محاكاة المدن الذكية. 


\section{(أمصادر}

[1] Mahmood, B., Tomasini, M., \& Menezes, R. (2015، October). Estimating memory requirements in wireless sensor networks using social tie strengths. In 2015 IEEE 40th Local Computer Networks Conference Workshops (LCN Workshops) (pp. 695-698). IEEE.

[2] Song, C., Koren، T., Wang، P., \& Barabási, A. L. (2010). Modelling the scaling properties of human mobility. Nature Physics, 6(10), 818.

[3] Tomasini, M., Zambonelli, F., Brayner, A., \& Menezes, R. (2013، September). Evaluating the performance of social networks of sensors under different mobility models. In 2013 International Conference on Social Computing (pp. 397-402). IEEE.

[4] Howard, A., Matarić, M. J., \& Sukhatme, G. S. (2002). Mobile sensor network deployment using potential fields: A distributed، scalable solution to the area coverage problem. In Distributed Autonomous Robotic Systems 5 (pp. 299-308). Springer، Tokyo.

[5] Aziz, A. A., Sekercioglu, Y. A., Fitzpatrick، P., \& Ivanovich, M. (2012). A survey on distributed topology control techniques for extending the lifetime of battery powered wireless sensor networks. IEEE communications surveys \& tutorials، 15(1), 121-144.

[6] Chellappan, S.، Gu, W., Bai, X., Xuan, D., Ma, B., \& Zhang, K. (2007). Deploying wireless sensor networks under limited mobility constraints. IEEE Transactions on Mobile Computing، 6(10), 1142-1157.

[7] de Melo, P. O. V., Viana, A. C.، Fiore, M., Jaffrès-Runser, K., Le Mouël, F., Loureiro, A. A., \& Guangshuo, C. (2015). RECAST: Telling apart social and random relationships in dynamic networks. Performance Evaluation، 87، 19-36.

[8] Mtibaa, A., May, M. ، Diot, C., \& Ammar, M. (2010، March). Peoplerank: Social opportunistic forwarding. In 2010 Proceedings IEEE INFOCOM (pp. 1-5). IEEE

[9] Mahmood B., Tomasini M., \& Menezes R. (2015). Social-driven information dissemination for mobile wireless sensor networks. Sensors \& Transducers, 189(6), 1-11.

[10] Tomasini, M. Zambonelli, F., \& Menezes, R. (2013، August). Using patterns of social dynamics in the design of social networks of sensors. In 2013 IEEE International Conference on Green Computing and Communications and IEEE Internet of Things and IEEE Cyber، Physical and Social Computing (pp. 685692). IEEE.

[11] Song, C., Qu, Z., Blumm, N., \& Barabási, A. L. (2010). Limits of predictability in human mobility. Science, 327(5968), 1018-1021. 
[12] Bandyopadhyay, S., Coyle, E. J., \& Falck, T. (2007). Stochastic properties of mobility models in mobile ad hoc networks. IEEE Transactions on Mobile Computing، 6(11), 1218-1229.

[13] Michelini, P. N.، \& Coyle, E. J. (2008، September). Mobility models based on correlated random walks. In Proceedings of the International Conference on Mobile Technology, Applications، and Systems (p. 86). ACM.

[14] Carpenter, J. R., \& Mashiku، A. K. (2015). Cauchy drag estimation for low earth orbiters.

[15] Mahmood, B., Tomasini، M.، Menezes, R., Social Network of Sensors، Netlogo Models, 2014.

[16] Sklar, E. (2007). NetLogo, a multi-agent simulation environment.

[17] Mahmood, B., \& Menezes, R. (2016). The Role of Human Relations and Interactions in Designing Memory-Related Models for Sensor Networks. Sensors \& Transducers, 199(4), 42-51. 